Portland State University

PDXScholar

\title{
A Study of Microwave curing of Underfill using Open and Closed microwave ovens
}

Aditya Thakare

Portland State University

Follow this and additional works at: https://pdxscholar.library.pdx.edu/open_access_etds

Part of the Other Computer Engineering Commons, and the Other Mechanical Engineering Commons Let us know how access to this document benefits you.

\section{Recommended Citation}

Thakare, Aditya, "A Study of Microwave curing of Underfill using Open and Closed microwave ovens" (2015). Dissertations and Theses. Paper 2246.

https://doi.org/10.15760/etd.2243

This Thesis is brought to you for free and open access. It has been accepted for inclusion in Dissertations and Theses by an authorized administrator of PDXScholar. Please contact us if we can make this document more accessible: pdxscholar@pdx.edu. 
A Study of Microwave Curing of Underfill using Open and Closed Microwave Ovens

by

Aditya Thakare

A thesis submitted in partial fulfillment of the requirements for the degree of

\author{
Master of Science \\ in \\ Mechanical Engineering
}

Thesis Committee:

Sung Yi, Chair

Faryar Etesami

Chien Wern

Portland State University

2015 


\begin{abstract}
As the demand for microprocessors is increasing with more and more consumers using integrated circuits in their daily life, the demand on the industry is increasing to ramp up production.
\end{abstract}

In order to speed up the manufacturing processes, new and novel approaches are trying to change certain aspects of it. Microwaves have been tried as an alternative to conventional ovens in the curing of the polymers used as underfills and encapsulants in integrated circuits packages. Microwaves however being electromagnetic waves have non uniform energy distribution in different settings, causing burning or incomplete cure of polymers.

In this study, we compare the two main types of microwaves proposed to perform the task of curing the polymers. To limit the study and obtain comparable results, both microwaves were limited to propagate in a single mode, TE10. The first is a closed microwave cavity using air as the propagation medium, and the second is an open microwave oven with a PTFE cavity that uses an evanescent field to provide energy.

The open air cavity was studied with different orientations of a substrate placed inside it so as to find the best case scenario in the curing process. This scenario was then compared with the best case scenario found for a sample cured in an evanescent field.

This comparison yielded results showing an advantage of the open microwave in maximum field present, thus leading to higher localized energy absorption and temperatures in the substrate, however this case also lead to a higher temperature 
gradient. The substrate cured in the closed microwave has a lower temperature gradient, but also a lower maximum field which leads to slower cure.

In the TE10 mode therefore, a closed microwave has an overall advantage as the heating process is only slightly slower than that of an open cavity, but the temperature gradient in this case is significantly lower. 


\section{Table of Contents}

Abstract

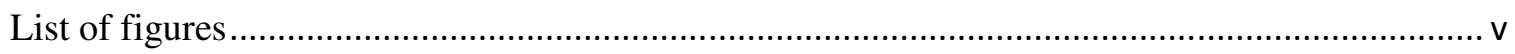

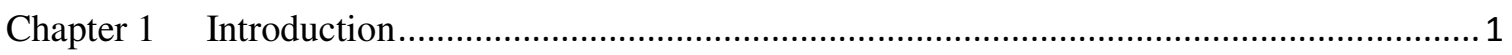

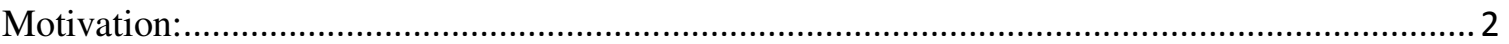

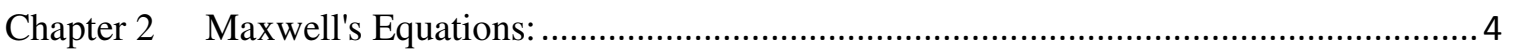

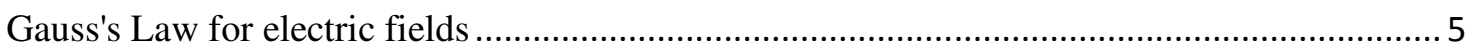

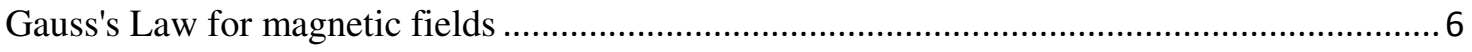

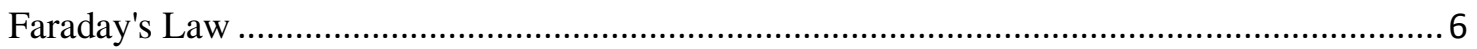

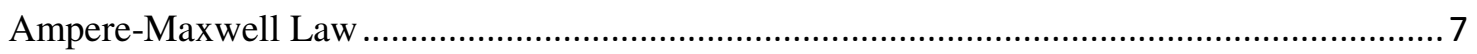

Chapter 3 Reflection inside a rectangular waveguide ....................................................... 9

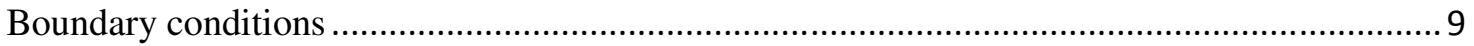

Transverse Electric (TE) transmission modes ................................................................... 10

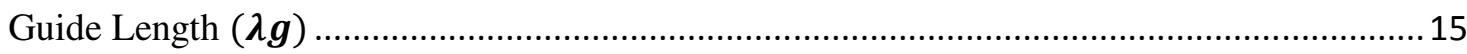

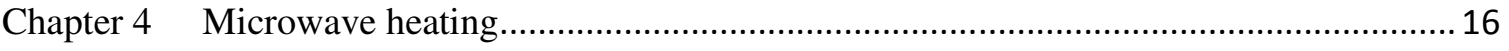

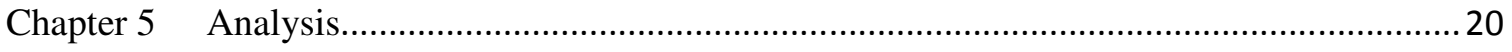

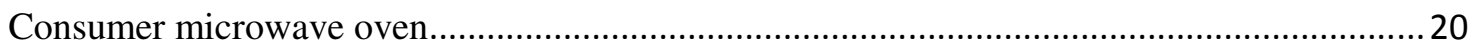

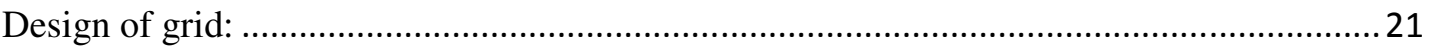

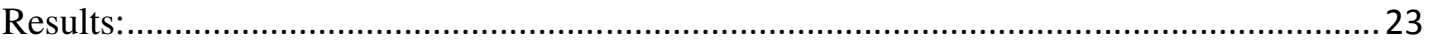

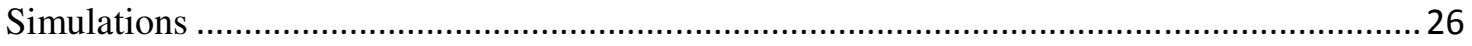

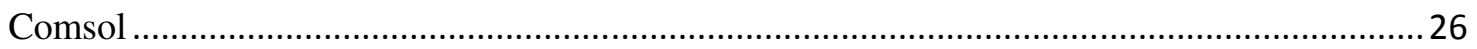

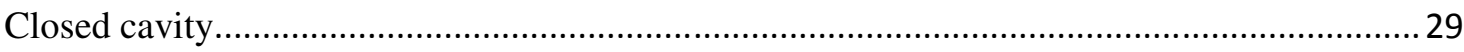

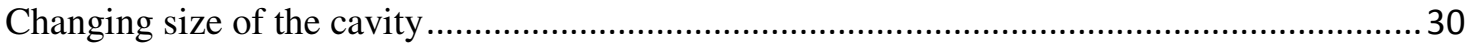

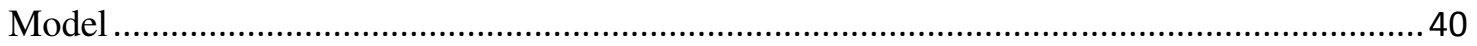

Position 1: Horizontal chip with centre of chip in centre of hotspot ..................................... 42

Position 2: Vertical chip with centre of chip at the centre of a hotspot .................................51

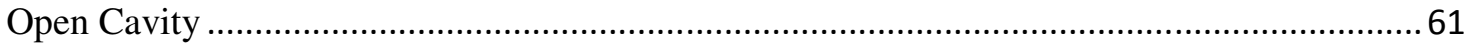

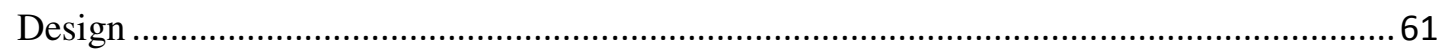

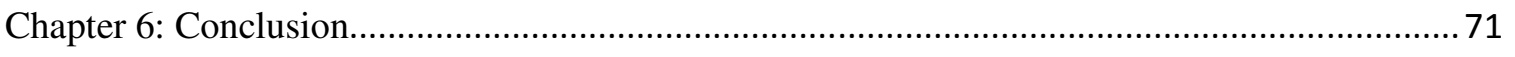

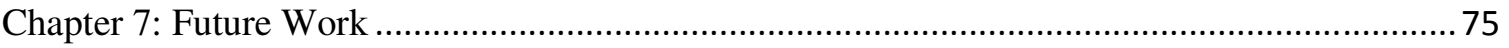




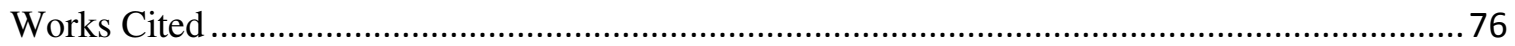

iv | P a g e 


\section{List of figures}

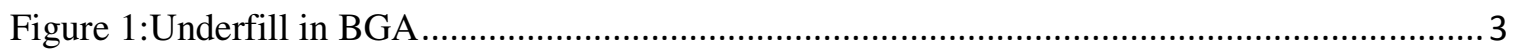

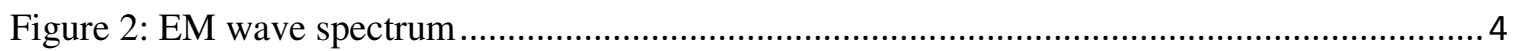

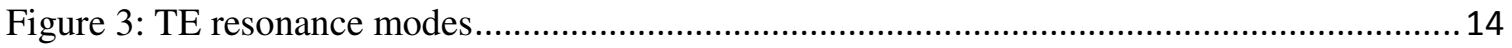

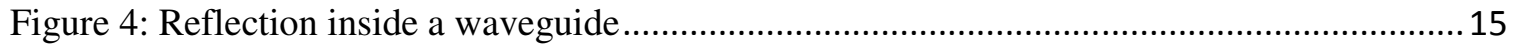

Figure 5: Permittivity changing with frequency ............................................................... 17

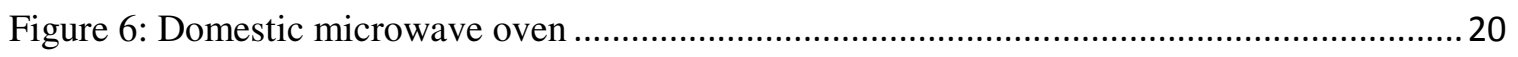

Figure 7: Grid used to measure temperature distribution....................................................... 21

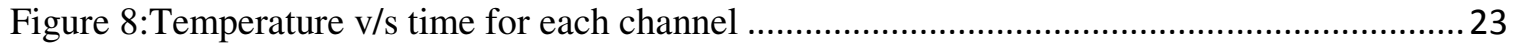

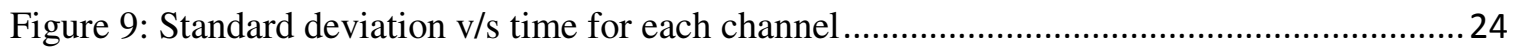

Figure 10: Temperature after 60 seconds of heating mapped on grid.........................................25

Figure 11: Electric field pattern in a regular sized cavity ........................................................ 31

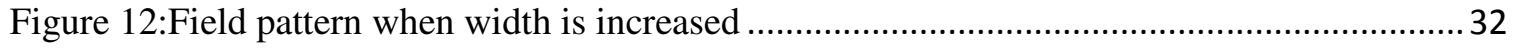

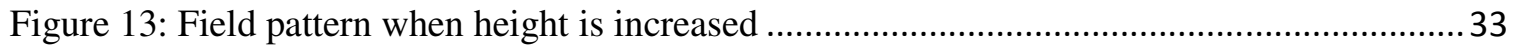

Figure 14: Field pattern for irregular geometry …................................................................... 34

Figure 15: Field as seen along the smaller(vertical) direction [Along 0 resonance direction] ....... 35

Figure 16: Field pattern after increasing height .................................................................... 36

Figure 17: Field pattern when height increased further ............................................................. 37

Figure 18: Field pattern after significant increase in height .................................................... 38

Figure 19: Model of standard waveguide with highlighted source ............................................. 41

Figure 20: Field pattern and horizontal substrate placement inside waveguide ........................... 42

Figure 21: Cut line showing measurement points across the cavity ........................................... 43

Figure 22: Electric field norm through the cavity in the presence of a horizontal substrate.......... 44

Figure 23:Electric field through the cavity in the presence of a horizontal substrate................... 45

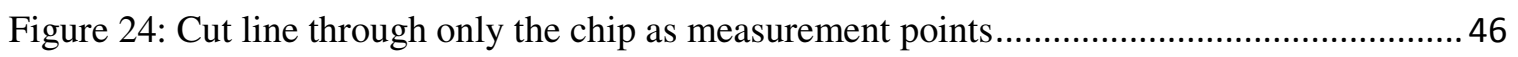

Figure 25: Electric field norm inside the horizontal chip ...................................................... 47

Figure 26: Temperature change in 0.1 second of heating ........................................................... 48

Figure 27: Temperature in the horizontal chip after 10 seconds of heating.................................49

Figure 28: Temperature inside the chip at 250s intervals ....................................................... 51

Figure 29: Field pattern and vertical substrate placement inside the waveguide..........................52

Figure 30: Cut line through the cavity to specify measurement points........................................53 
Figure 31: Electric field norm through the cavity in the presence of a vertical chip.....

Figure 32: Electric field norm inside the chip placed vertically in the cavity ..............................55

Figure 33:Temperature in the substrate after 10s of heating..................................................... 56

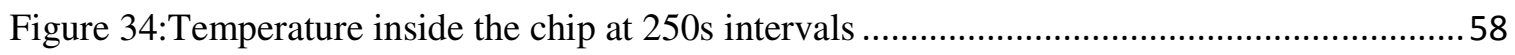

Figure 35: Electric field norm inside the vertical chip with changing thickness ..........................59

Figure 36: Electric field norm inside a horizontal chip with changing thickness .........................60

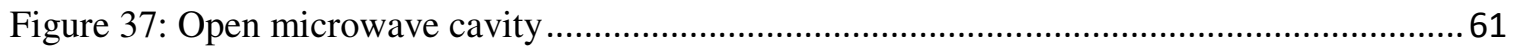

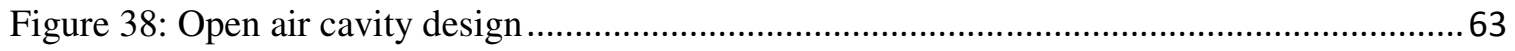

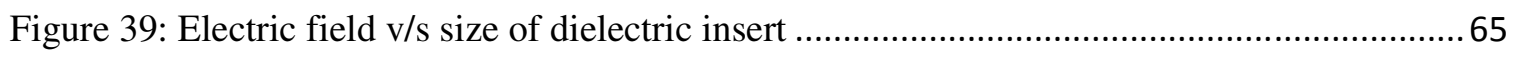

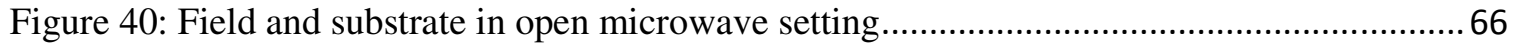

Figure 41: Cut line through the chip showing measurement points...........................................67

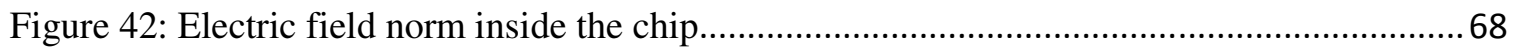

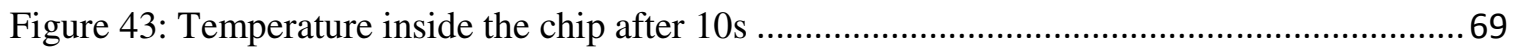

Figure 44: Temperature inside the chip at 250s intervals ........................................................ 70

Figure 45: Maximum temperature inside each chip.................................................................... 71

Figure 46: Maximum temperature gradient inside each chip................................................... 73 


\section{Chapter $1 \quad$ Introduction}

Microwaves in their modern interpretation as a source of energy for heating objects were discovered by Percy Spencer, by accident during research for radar tube design in 1939 . (Hiskey) This technology has since been used at a large scale in commercial microwave ovens used to cook and warm food since the patent for the microwave cooking oven was filed in 1945 (Hiskey). The use of microwaves to cure the polymers used in underfill and as encapsulants for integrated circuits however, is a recent trend.

The polymers used in the encapsulants and underfill are a variety of thermosetting polymers that are in a liquid or gel state when in their uncured or monomeric state. Conventionally, diffusion and laminar flow based convection ovens have been used to cure these thermosets. This is a bottleneck in the production of IC packages in this day and age as the processing time in the ovens takes several hours. Microwave curing of polymers on the other hand has been attained in the order of a few minutes. (Sumanth Kumar Pavuluri, 2012)

Replacing the convection ovens with microwaves therefore would speed up the production time by close to $90 \%$. This is achieved due to the fact that microwaves can penetrate the these polymers and provide energy for the curing process without depending solely on conductive heat as its only energy source. In certain cases, polymers cured using microwaves have also been known to have better properties as compared to their thermally cured counterparts. (Eva Marand, 1992) 
A typical microchip using ball grid array (BGA) technology that requires an underfill has dimensions of about $23 \mathrm{~mm}$ per side with a height of about $1 \mathrm{~mm}$. (Flip Chip Ball Grid Array Package Reference Guide, 2005) Assuming a rather large chip in a maximum size case, this document explores the absorption patterns of a $40 \times 40 \times 1 \mathrm{~mm}$ chip using two different types of microwave ovens. (Zak Fathi)

Several advantages of curing polymers using microwaves have been observed. Increased polymerization rate of epoxy curing, reduced drying time, increased glass transition temperature, and increased strength are all known to have occur in polymers cured in this fashion. (Martin C. Hawley) These advantages make microwave curing a very appealing idea in the industry and is therefore a topic worth exploring.

\section{Motivation:}

The semiconductor industry has been steadily moving towards smaller and smaller package sizes. These higher densities require high density packaging designs such as flip

chip packaging. These designs however undergo stresses in the solder balls when the chip is used as thermal fatigue cycles are very prevalent in operating conditions of these ICs. The different materials in the chip that heat up during usage have different coefficients of thermal expansion. Due to this mismatch in the coefficient of thermal expansion of the materials of the chip and board, measures have to be taken to ensure that the solder joints do not break due to the fatigue stresses induced. 

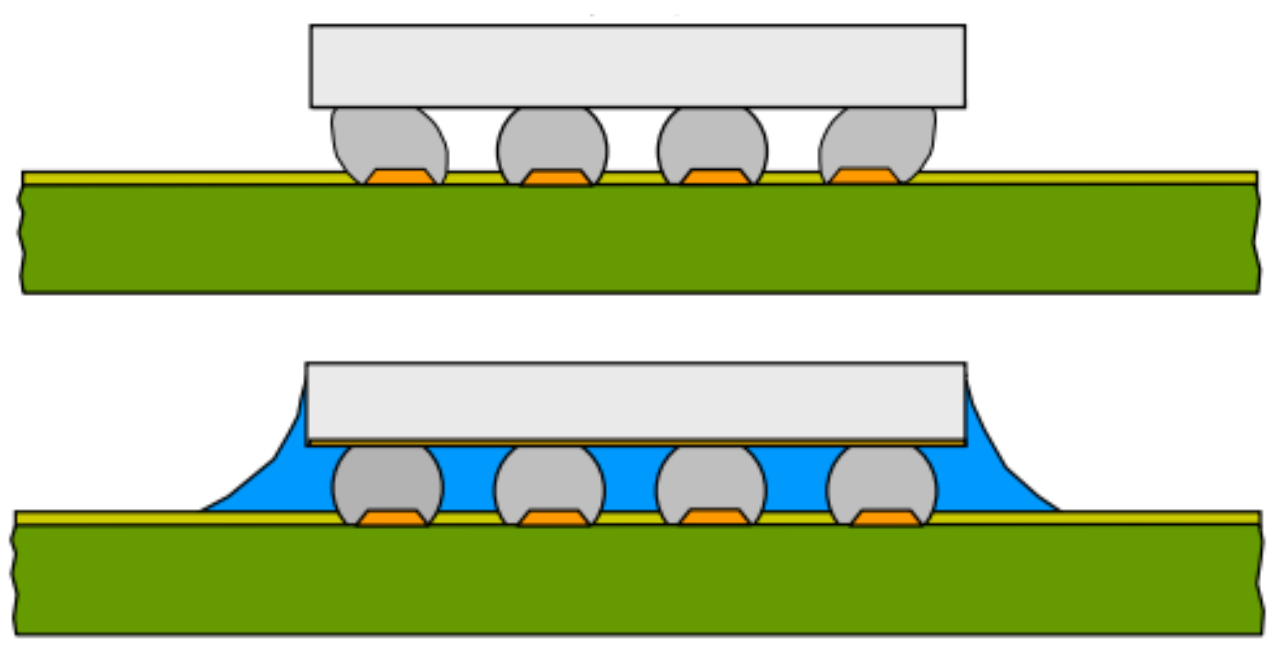

Figure 1:Underfill in BGA

In order to minimize these stresses, the solder balls are covered with a polymeric material (Johnson) and form a more reliable electronic package. These polymers are often used to encapsulate the entire chip so as to protect the chip. The curing of these polymers is one of the slowest processes in the total manufacturing time of the package and therefore alternative curing methods must be explored. 


\section{Chapter 2 Maxwell's Equations:}

In order to understand the power distribution within the microwave cavity, we first need to understand the nature of the electromagnetic radiation that microwaves are.

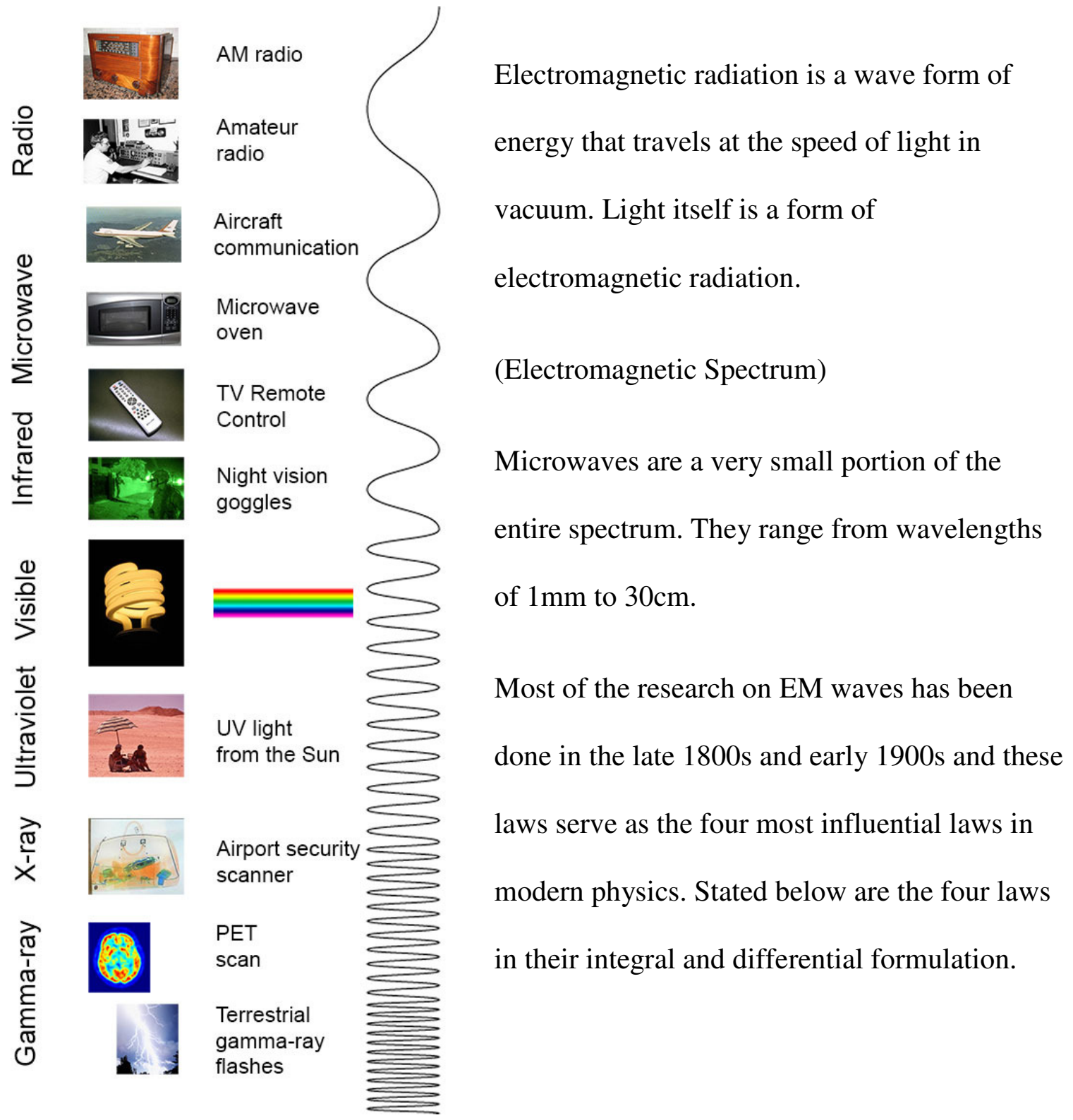

Figure 2: EM wave spectrum 


\section{Gauss's Law for electric fields}

This law deals with the electrostatic fields. In the integral formulation, the law states that:

Electric charge produces an electric field, and the flux of that field passing through any closed surface is proportional to the total charge contained within that field.

$$
\oint_{S} \vec{E} \cdot \hat{n} d a=\frac{q_{e n c}}{\varepsilon_{0}}
$$

where $\vec{E}$ is the electric field in Newtons per Coulomb $[N / C]$

$q_{\text {enc }}$ is the (enclosed) charge in Coulombs $[C]$

$\varepsilon_{0}$ is the electric permittivity of free space

In the differential form, the law can be written as:

The electric field produced by electric charge diverges from positive charge and converges upon negative charge.

$$
\vec{\nabla} \cdot \vec{E}=\frac{\rho}{\varepsilon_{0}}
$$

where $\rho$ is the charge density in Coulombs per cubic meter $\left[C / \mathrm{m}^{3}\right]$ 
Gauss's Law for magnetic fields

The law in its integral formulation states that:

The total magnetic flux passing through any closed surface is zero.

$$
\oint_{S} \vec{B} \cdot \hat{n} d a=0
$$

where $\vec{B}$ is the magnetic field in Teslas [T]

In its differential form, it is simplified to:

The divergence of the magnetic field at any point is zero

$$
\vec{\nabla} \cdot \vec{B}=0
$$

\section{Faraday's Law}

In its integral formulation, this law states that:

Changing magnetic flux through a surface induces an electromotive force in any boundary path of that surface, and a changing magnetic field induces a circulating electric field.

$$
\oint_{C} \vec{E} \cdot \widehat{d l}=\frac{d}{d t} \int_{S} \vec{B} \cdot \hat{n} d a
$$


This gets simplified in the differential form to:

A circulating electric field is produced by a magnetic field that changes with time.

$$
\vec{\nabla} \times \vec{E}=\frac{\partial \vec{B}}{\partial t}
$$

\section{Ampere-Maxwell Law}

In its integral notation, this law states that:

An electric current or a changing electric flux through a surface produces a circulating magnetic field around any path that bounds that surface.

$$
\oint_{C} \vec{B} \cdot \overrightarrow{d l}=\mu_{0}\left(I_{e n c}+\varepsilon_{0} \frac{d}{d t} \int_{S} \vec{E} \cdot \hat{n} d a\right)
$$

where: $\mu_{0}$ is the magnetic permeability of free space

$$
I_{e n c} \text { is enclosed electric current in Amperes[A] }
$$

Whereas in the differential form, it states that:

A circulating magnetic field is produced by an electric current and by an electric field that changes with time.

$$
\vec{\nabla} \times \vec{B}=\mu_{0}\left(\vec{J}+\varepsilon_{0} \frac{\partial \vec{E}}{\partial t}\right)
$$


where: $\vec{J}$ isthe electric current density in Amperes per square meter $\left[A / \mathrm{m}^{2}\right]$ (Fleisch, 2008)

The reason the four laws stated above are called Maxwell's equations is due to the fact that he combined them into a single theory of electromagnetism. This culminated in the formulation of the wave equation. Shown below are wave equation with the electric and magnetic field respectively. (Fleisch, 2008)

$$
\begin{gathered}
\nabla^{2} \vec{E}=\mu_{0} \varepsilon_{0} \frac{\partial^{2} \vec{E}}{\partial t^{2}} \\
\nabla^{2} \vec{B}=\mu_{0} \varepsilon_{0} \frac{\partial^{2} \vec{B}}{\partial t^{2}}
\end{gathered}
$$




\section{Chapter $3 \quad$ Reflection inside a rectangular waveguide}

In order to understand the energy distribution inside a cavity, we must first understand how these waves travel inside a waveguide. Since the wave propagating inside the waveguide is a resonance pattern of the wave entering the guide from the source, we must understand how the propagating wave front comes about.

Waveguides are generally made of a metallic material. This is due to the fact that no electric fields exist in an ideal conductor. Thus implying that incident EM radiation is reflected at the perfect conductor's surface.

\section{Boundary conditions}

Using Maxwell's equations at the interactions of electromagnetic waves trying to enter a conductive medium from a non conductive medium, we can get 4 boundary conditions, one from each equation.

$$
\begin{gathered}
E_{n}=\frac{\rho_{s}}{\varepsilon_{0}} \\
E_{t}=0 \\
B_{n}=0 \\
\left|B_{t}\right|=\mu_{0}\left|J_{s}\right|
\end{gathered}
$$

(Mavalvala)

These boundary conditions when implemented on a rectangular waveguide allow us to understand the reflection patterns inside. Since due to this reflection pattern, the wave interacts with itself, this culminates in the formation of transmission modes. 


\section{Transverse Electric (TE) transmission modes}

In order to calculate the reflection pattern or transmission modes inside a waveguide, let us assume a rectangular waveguide of dimensions $a$ and $b$ filled with a material having permittivity $\varepsilon$ and permeability $\mu$.

Let the longer side of the waveguide be aligned with the x-axis such that $a>b$

TE propagation is characterized by fields with $E_{z}=0$

This implies that $H_{z}$ must satisfy the reduced wave equation:

$$
\left(\frac{\partial^{2}}{\partial x^{2}}+\frac{\partial^{2}}{\partial y^{2}}+k_{c}^{2}\right) h_{z}(x, y)=0
$$

Where $H_{z}(x, y, z)=h_{z}(x, y) e^{-j \beta z}$ and $k_{c}=\sqrt{k^{2}-\beta^{2}}$ is the cutoff wave number

$$
\text { and } k=\frac{2 \pi}{\lambda}
$$

Using separation of variables,

$$
h_{z}(x, y)=X(x) Y(y)
$$

We can obtain that

$$
\frac{1}{X} \frac{d^{2} X}{d x^{2}}+\frac{1}{Y} \frac{d^{2} Y}{d y^{2}}+k_{c}^{2}=0
$$

This leads to

$$
\frac{d^{2} X}{d x^{2}}+k_{x}^{2} X=0
$$




$$
\begin{aligned}
& \frac{d^{2} Y}{d y^{2}}+k_{y}{ }^{2} Y=0 \\
& k_{x}{ }^{2}+{k_{y}}^{2}=k_{c}{ }^{2}
\end{aligned}
$$

This approach yields a general solution of

$$
h_{z}(x, y)=\left(A \cos _{x} x+B \sin _{x} x\right)\left(C \cos k_{y} y+D \sin k_{y} y\right)
$$

Implementing the boundary conditions:

$$
\begin{aligned}
& e_{x}(x, y)=0, \text { at } y=0, b \\
& e_{y}(x, y)=0, \text { at } x=0, a
\end{aligned}
$$

In the equations to be used:

$$
\begin{gathered}
e_{x}=\frac{-j \omega \mu}{k_{c}{ }^{2}} k_{y}\left(A \cos k_{x} x+B \sin k_{x} x\right)\left(-C \sin k_{y} y+D \cos k_{y} y\right) \\
e_{y}=\frac{j \omega \mu}{k_{c}^{2}} k_{x}\left(-A \sin k_{x} x+B \cos k_{x} x\right)\left(C \cos k_{y} y+D \sin k_{y} y\right)
\end{gathered}
$$

When the boundary conditions are applied, we get that $D=0$, and $k_{y}=\frac{n \pi}{b}$ for $n=$ $0,1,2 \ldots$

$B=0$ and $k_{x}=\frac{m \pi}{a}$ for $m=0,1,2 \ldots$

Here $m$ and $n$ are the modes of transmission in their respective dimensions

This leads to:

$$
H_{z}(x, y, z)=A_{m n} \cos \frac{m \pi x}{a} \cos \frac{n \pi y}{b} e^{-j \beta z}
$$

Where $A_{m n}$ is an arbitrary amplitude constant consisting of the constants $A$ and $C$ 
The transverse field components of the field in TE mode can therefore be summarized

as:

$$
\begin{gathered}
E_{x}=\frac{j \omega \mu n \pi}{k_{c}^{2} b} A_{m n} \cos \frac{m \pi x}{a} \sin \frac{n \pi y}{b} e^{-j \beta z} \\
E_{y}=\frac{-j \omega \mu m \pi}{k_{c}^{2} a} A_{m n} \sin \frac{m \pi x}{a} \cos \frac{n \pi y}{b} e^{-j \beta z} \\
H_{x}=\frac{j \beta m \pi}{k_{c}^{2} a} A_{m n} \sin \frac{m \pi x}{a} \cos \frac{n \pi y}{b} e^{-j \beta z} \\
H_{y}=\frac{j \beta n \pi}{k_{c}^{2} b} A_{m n} \cos \frac{m \pi x}{a} \sin \frac{n \pi y}{b} e^{-j \beta z}
\end{gathered}
$$

Where the propagation constant is:

$$
\beta=\sqrt{k^{2}-k_{c}^{2}}=\sqrt{k^{2}-\left(\frac{m \pi}{a}\right)^{2}-\left(\frac{n \pi}{b}\right)^{2}}
$$

which is real, corresponding to a propagation mode when:

$$
k>k_{c}=\sqrt{\left(\frac{m \pi}{a}\right)^{2}+\left(\frac{n \pi}{b}\right)^{2}}
$$

The cutoff frequency is therefore given by

$$
f_{c_{m n}}=\frac{k_{c}}{2 \pi \sqrt{\mu \varepsilon}}=\frac{1}{2 \pi \sqrt{\mu \varepsilon}} \sqrt{\left(\frac{m \pi}{a}\right)^{2}+\left(\frac{n \pi}{b}\right)^{2}}
$$

or

$$
f_{c, m n}=\frac{c}{2 \pi \sqrt{\mu_{r} \varepsilon_{r}}} \sqrt{\left(\frac{m \pi}{a}\right)^{2}+\left(\frac{n \pi}{b}\right)^{2}}
$$


can be simplified to

$$
f_{c, m n}=\frac{c_{m}}{2} \sqrt{\left(\frac{m}{a}\right)^{2}+\left(\frac{n}{b}\right)^{2}}
$$

where $c_{m}$ is the speed of light in the propagating medium.

In the above cases, $\mathrm{m}$ and $\mathrm{n}$ stand for the oscillation modes in the cross sectional cavity, perpendicular to the direction of propagation. Examples of how the field lines look inside the waveguide for different propagation modes are shown below. (Pozar, 2012) 

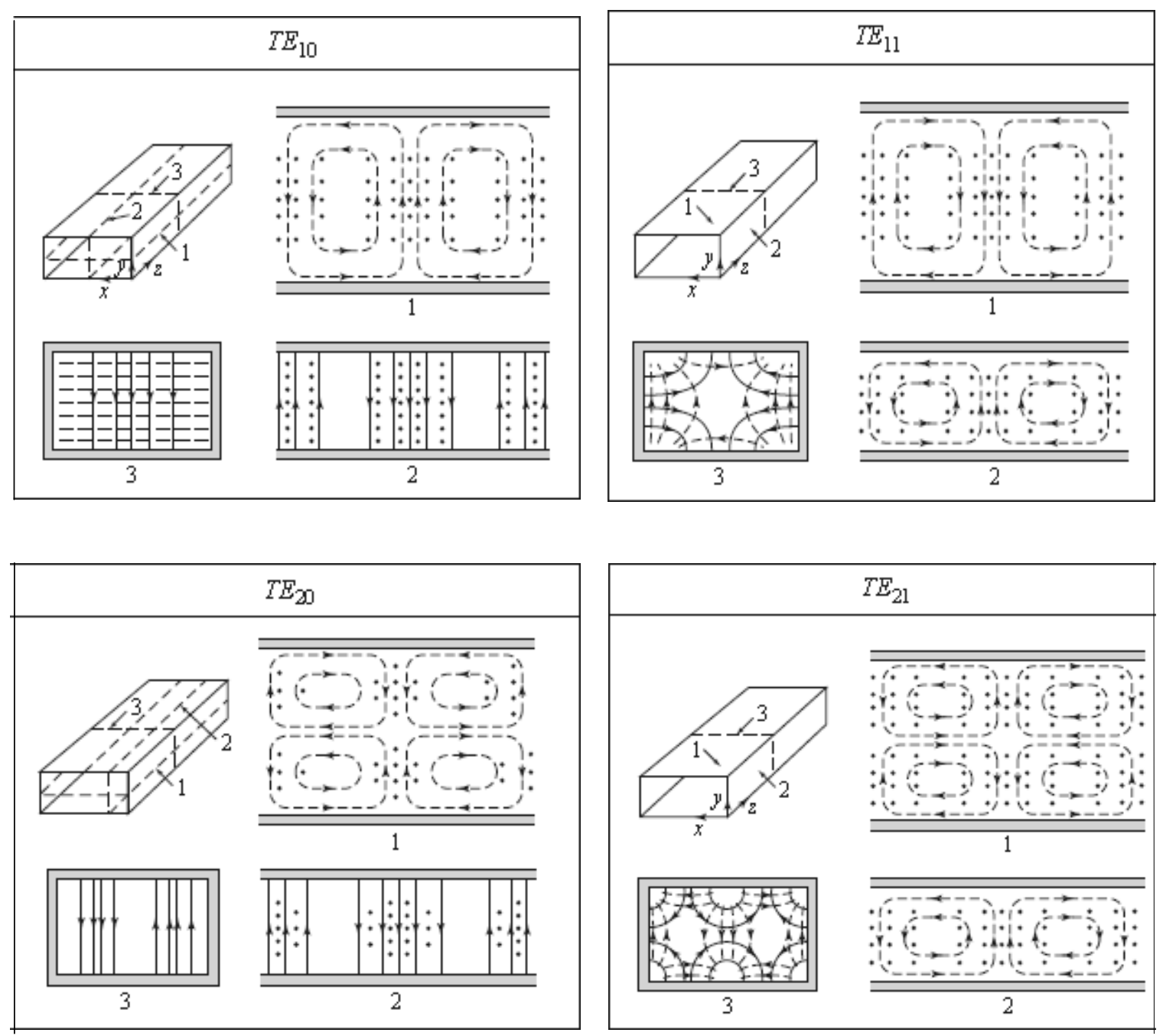

Figure 3: TE resonance modes

Through this analysis, we have limited the investigation to the most fundamental mode of TE10. For this mode, the cutoff frequency within the waveguide is given by:

$$
f_{c_{10}}=\frac{1}{2 a \sqrt{\mu \varepsilon}}
$$

or

$$
f_{c_{10}}=\frac{c_{m}}{2 a}
$$

where $c_{m}$ is the speed of light in the given medium 


\section{Guide Length $\left(\lambda_{g}\right)$}

The guide length is defined as the propagating wavelength of the wave front inside the cavity. This wavelength is different from the wavelength of the radiation emitted by the source as the propagating wave in the cavity is an interference pattern of the wave with itself reflecting off the walls of the waveguide. (Staelin) (Mavalvala)

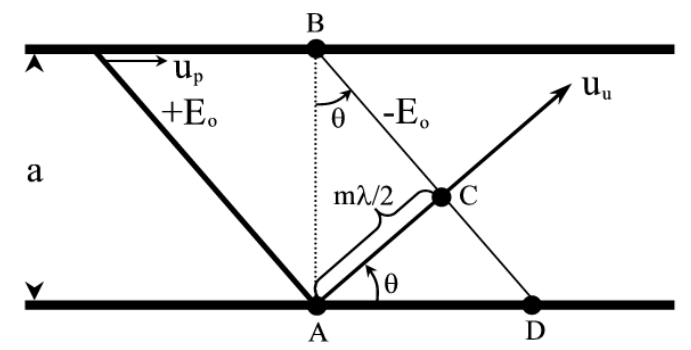

Figure 4: Reflection inside a waveguide

The guide length is given by

$$
\begin{gathered}
\lambda_{g}=\frac{2 \pi}{\beta}>\frac{2 \pi}{k}=\lambda \\
\lambda_{g}=\frac{2 \pi}{\sqrt{k^{2}-\left(\frac{m \pi}{a}\right)^{2}-\left(\frac{n \pi}{b}\right)^{2}}}
\end{gathered}
$$




\section{Chapter $4 \quad$ Microwave heating}

Microwave heating, also called dielectric heating is a form of heating that occurs due to the interaction of an oscillating electric field with polar groups of non conducting molecules.

In the presence of an electric field, the groups align themselves with the field by undergoing polarization given by:

$$
\overrightarrow{\boldsymbol{P}}=\left(\varepsilon_{0}^{\prime}-1\right) \varepsilon_{0} \overrightarrow{\boldsymbol{E}}=\overrightarrow{\boldsymbol{D}}-\varepsilon_{0} \overrightarrow{\boldsymbol{E}}
$$

Where: $\overrightarrow{\boldsymbol{E}}=$ electric field vector

$$
\begin{aligned}
& \overrightarrow{\boldsymbol{P}}=\text { polarization vector } \\
& \overrightarrow{\boldsymbol{D}}=\text { dielectric displacement vector }
\end{aligned}
$$

In an oscillating field, the dielectric constant has a complex value due to the dielectric displacement $(\overrightarrow{\boldsymbol{D}})$ being out of phase with the electric field $(\overrightarrow{\boldsymbol{E}})$ with

$$
\varepsilon *=\varepsilon_{r}^{\prime}-j \varepsilon_{r}^{\prime \prime}
$$

This allows us to obtain the loss factor of

$$
\tan (\delta)=\varepsilon_{r}^{\prime \prime} / \varepsilon_{r}{ }^{\prime}
$$

where $\varepsilon_{r}^{\prime \prime}$ and $\varepsilon_{r}^{\prime}$ are usually temperature and frequency dependent 
They depend on the atomic and molecular structure of the given compound, although $\varepsilon_{r}{ }^{\prime \prime}$ has maximum values at certain frequency ranges around the natural frequency of the material $\left(\omega_{m}\right)$ and $\varepsilon_{r}{ }^{\prime}$ decreases in said frequency range by $\Delta \varepsilon_{r}{ }^{\prime}$

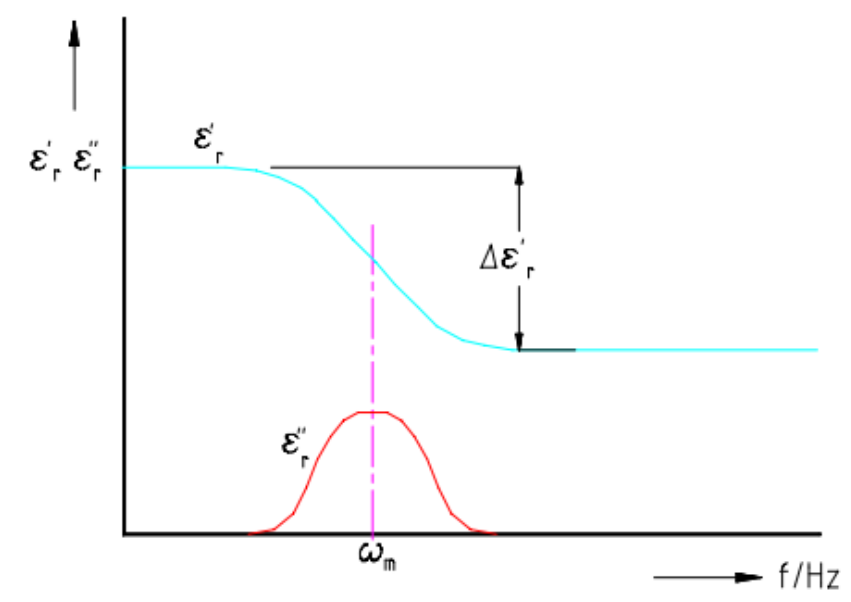

Figure 5: Permittivity changing with frequency

Understanding the heating phenomenon, we apply Maxwell's equations to the free dielectric material.

Given a volume $(\mathrm{V})$ and the surface $(\mathrm{F})$ with dielectric constant $(\varepsilon)$ and permiability $(\mu)$ which are constant in time and is free of electric charges

$$
\begin{gathered}
\nabla D=0 \\
\nabla B=0 \\
\nabla \times E=-j \omega \mu_{0} \mu_{r} H \\
\nabla \times H=\left(k+\omega \varepsilon_{0} \varepsilon\right) E
\end{gathered}
$$


The conductivity $(\mathrm{k})$ of the material comes into a significant role along with the dielectric constant $\varepsilon_{r}{ }^{\prime}$ due to the material being a non conductor and therefore producing displacement currents which can not be neglected.

$$
\nabla \times H=j \omega \varepsilon_{r}^{\prime} \varepsilon_{0}\left(1+\frac{k}{\omega \varepsilon_{r}^{\prime} \varepsilon_{0}}\right) E=j \omega \varepsilon * E
$$

with $\varepsilon *=\varepsilon_{r}^{\prime} \varepsilon_{0}\left(1+\frac{k}{\omega \varepsilon_{r}^{\prime} \varepsilon_{0}}\right)$

Converting the volume integral of $\nabla(E \times H)$ to an area integral using the Gaussian equation, we obtain:

$$
\int_{F} \nabla(E \times H) d F=-j \omega \int_{V} \mu(H H *) d V+j \omega \int_{V} \varepsilon *(E E *) d V
$$

To calculate activity losses, we calculate the energy converted to heat in a given unit of time for a given volume:

$$
W=\int_{F}(E \times H) d F=\int_{F} \mathrm{~S} d F
$$

Where $\mathrm{S}$ is the irradiation vector that indicates the amount of energy flow in a point of space.

this gives the energy loss as

$$
P=k \int(E E *) d V
$$


Where $k=\omega \varepsilon_{r}^{\prime \prime} \varepsilon_{0}=\omega \varepsilon_{r}^{\prime} \varepsilon_{0} \tan \delta$

Thus

$$
P_{\text {loss }}=\omega \varepsilon_{r}^{\prime \prime} \varepsilon_{0} \int|E|^{2} d V
$$

(Bremen, 1997) (Metaxas, 1991)

We can see through this that $P_{\text {loss }} \propto|E|^{2}$ allowing us to understand how the power trend will exist inside the substrate placed inside the EM field. 


\section{Chapter $5 \quad$ Analysis}

The goal of this analysis is to attain the minimum possible temperature gradient within the sample substrate that is to be cured using microwaves. The start of the investigation was the exploration of the energy distribution and resultant temperature distribution inside a domestic microwave oven.

\section{Consumer microwave oven}

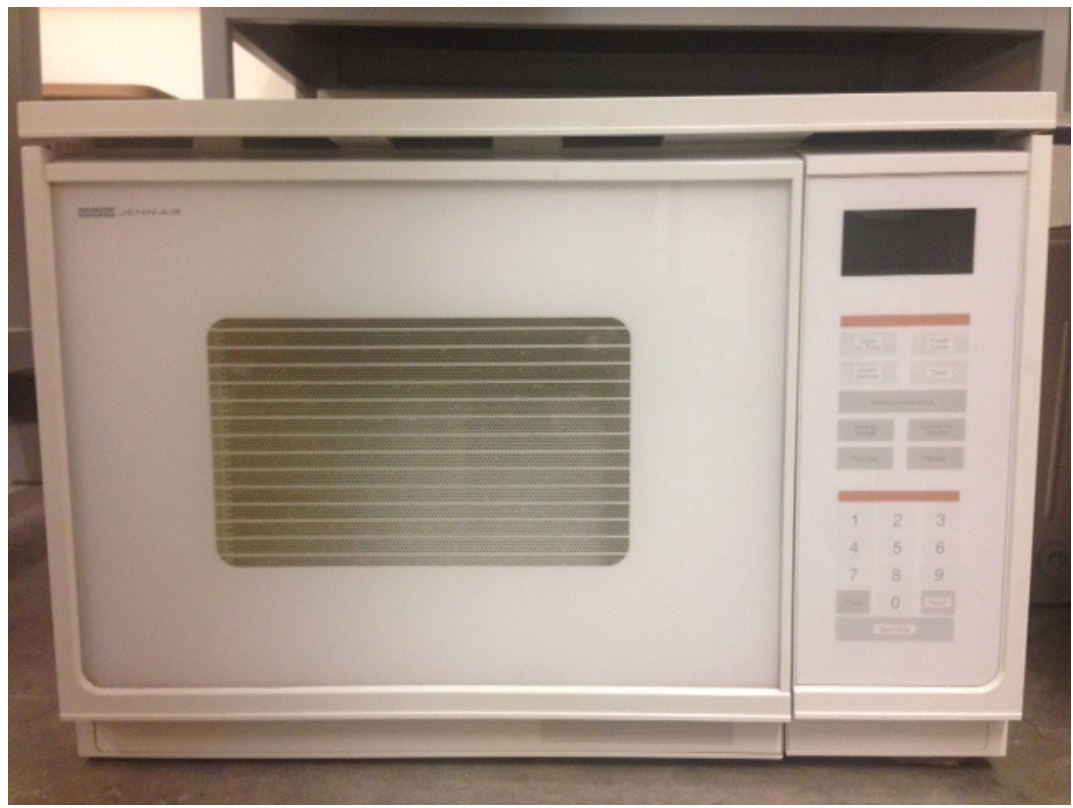

In an ideal case, the microwave cavity would have a completely even energy distribution, which in turn would lead to a completely even temperature

Figure 6: Domestic microwave oven distribution inside the substrate.

This however is not possible due to the fact of the microwaves used are electromagnetic waves, and in this case with a fixed frequency, and therefore have a fixed wavelength. These electromagnetic waves bounce around the cavity and form standing waves. The size and shape of these standing waves is dependent on the excitation frequency and dimensions of the waveguide as discussed in chapter 3. 
In order to understand the standing pattern created in the cavity, an experiment was designed to measure the temperature distribution in a substrate placed in the cavity, which in turn would allow us to predict the energy distribution inside the cavity, localized around the substrate placed within it.

In order to measure the temperature inside the cavity, a grid was developed with columns of fixed size containing water as a replacement for the substrate. Thermocouples were used to measure the temperature of this water in real time while it was being heated inside the microwave cavity.

\section{Design of grid:}

A $20 \mathrm{~cm} \times 20 \mathrm{~cm}$ block was machined so as to create a $4 \times 4$ grid of pockets to hold a $1 \mathrm{~cm}$ depth of water column.

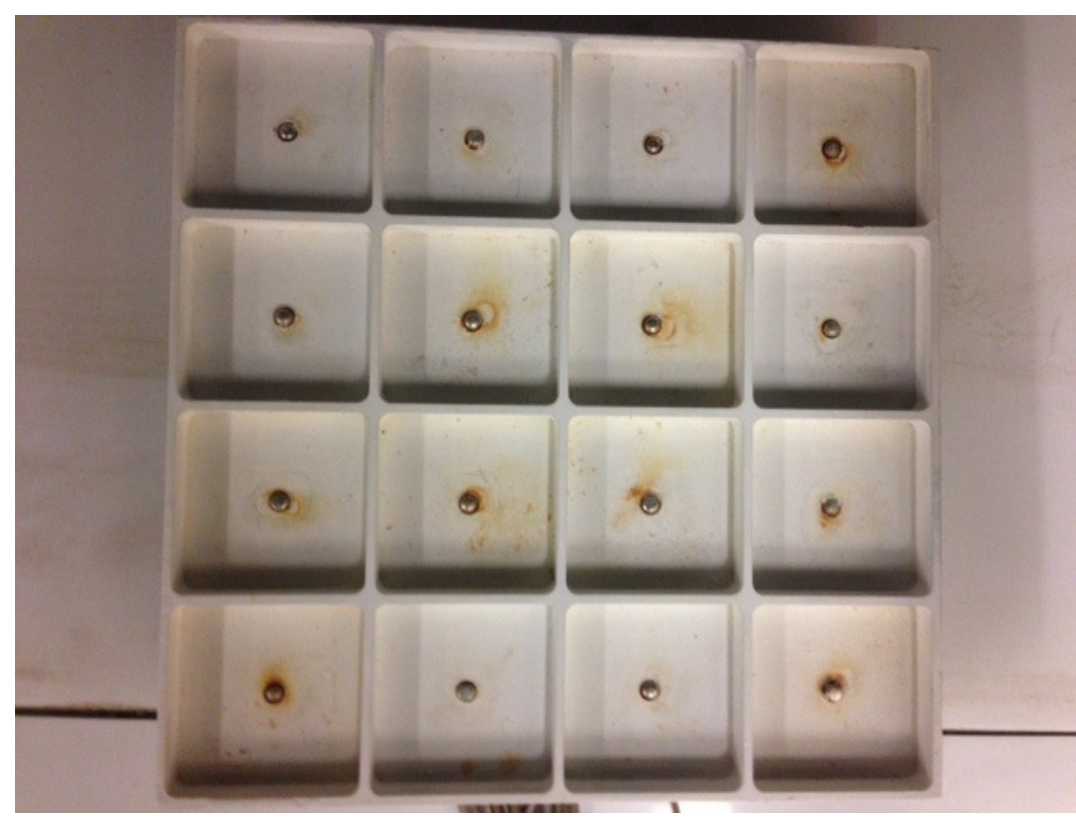

Figure 7: Grid used to measure temperature distribution
This leads to a chamber size smaller than the wavelength of the radiation and therefore should not cause errors due to interaction with the waves.

K- type thermocouples were placed at the

centre of each column from underneath so that the temperature of the water could be 
measured without interacting with the microwaves. The thermocouples were connected to a digital to analog converter (DAC), in this case an NI-6210 in order to retrieve the data. The DAC was operated using Labview to collect data and record it for further usage.

Several runs were conducted, each measuring the heating up of the sample for one minute. This allowed us to obtain statistically significant results that could be analyzed. 


\section{Results:}

The temperature in every column increased with time, however certain columns were heated more than others. The temperatures and standard-deviations for all the runs are shown below.

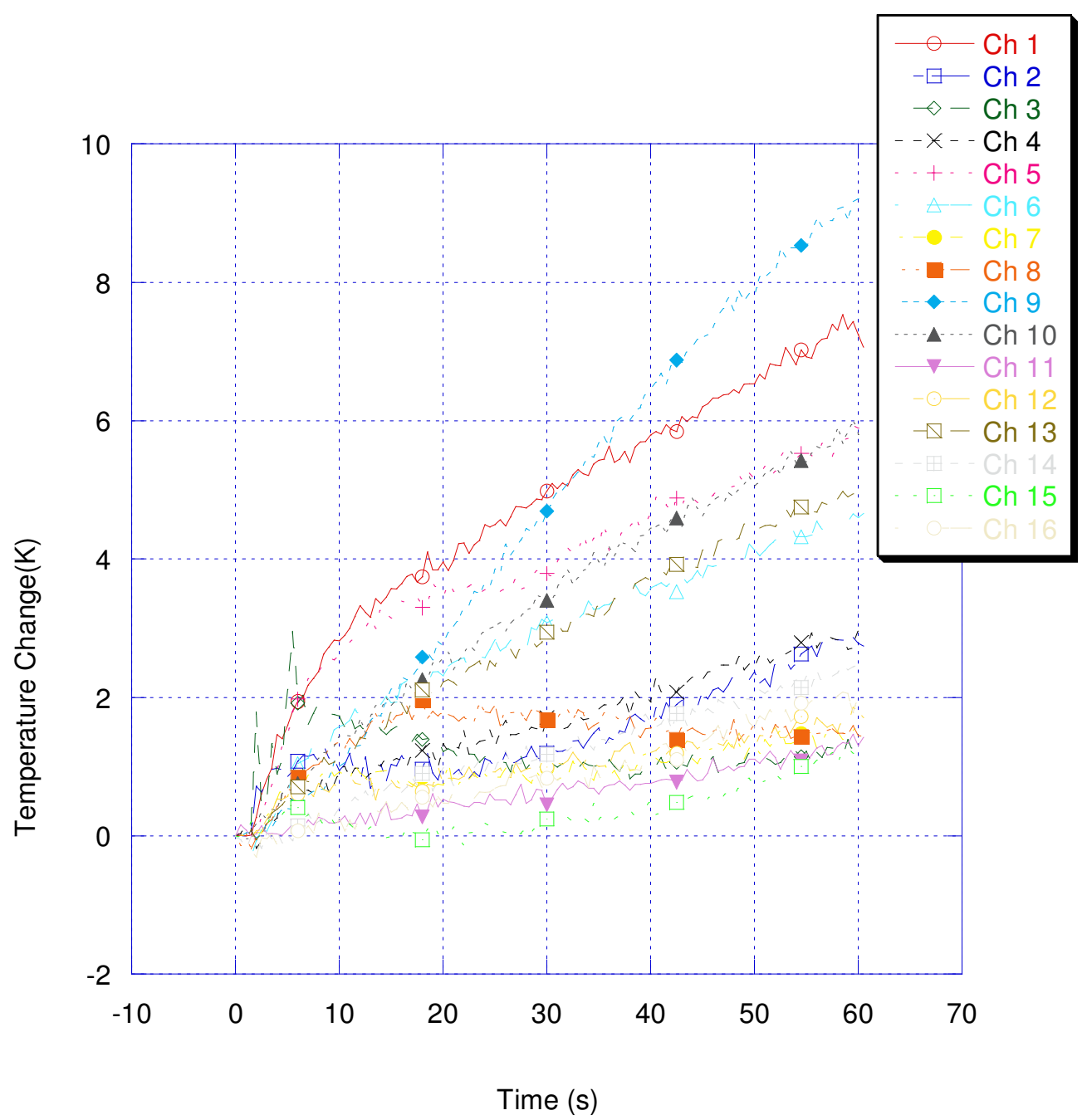

Figure 8:Temperature v/s time for each channel 


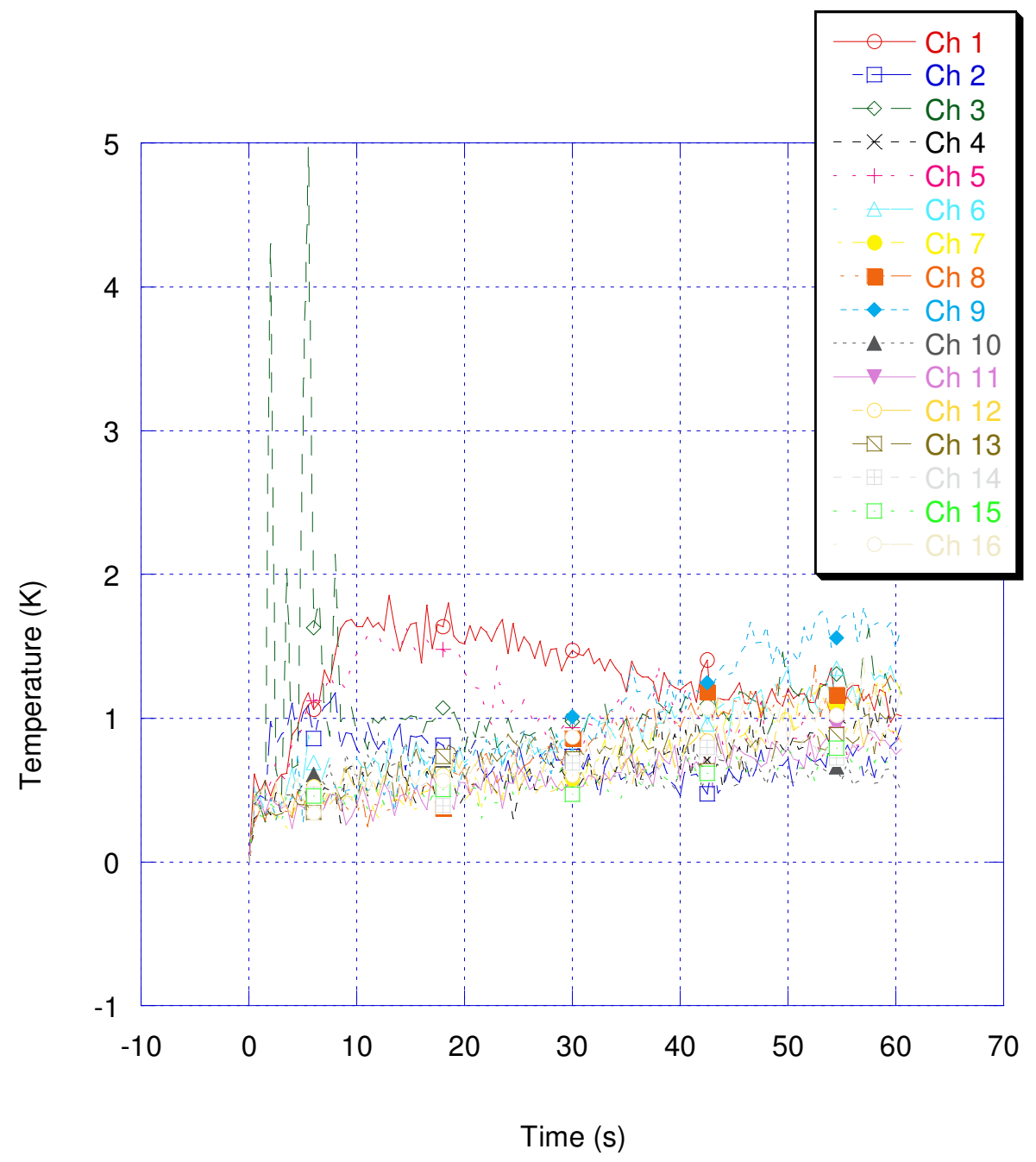

Figure 9: Standard deviation v/s time for each channel

The erratic behavior of one of the thermocouples is due to interactions with the microwaves that leaked into the chamber housing the thermocouples. The large and sudden change is the standard deviation of channel 3 shows the effects of this leakage. Though several steps were taken to prevent leakage, the small amount of microwaves that leaked through were able to cause the noise in the readings. 
Placing this data on the grid designed so as to visualize it, we see the following distribution inside the microwave oven cavity after one minute of heating:

\begin{tabular}{|c|c|c|c|c|}
\hline \multirow{4}{*}{ શి E } & 7.07 & 2.74 & 1.16 & 2.90 \\
\hline & 5.73 & 4.66 & 1.52 & 1.42 \\
\hline & 9.24 & 5.81 & 1.43 & 1.70 \\
\hline & 4.90 & 2.5 & 1.20 & 2.03 \\
\hline
\end{tabular}

Figure 10: Temperature after 60 seconds of heating mapped on grid Looking at the Temperatures of the various parts in this grid, we can see that the maxima comes about every 2 columns, or about every $10 \mathrm{~cm}$. This gives a starting point of what one may expect when trying to calculate for the distribution of energy inside a microwave cavity.

To confirm these results, simulations of other large cavity microwaves were conducted where excitation was still conducted by the same waveguide and frequency so as to compare the results observed and gain a better understanding. 


\section{Simulations}

In order to mimic the physical model, the design for this analysis was limited to a single frequency microwave generator transmitting at a single mode. In order to achieve this, the frequency was first confirmed and restricted to $2.45 \mathrm{GHz}$. This is the frequency used by domestic microwaves and is relatively cheap and easy to obtain. The propagation modes were also limited to the fundamental mode, TE10 which is the same mode used by domestic microwave ovens.

\section{Comsol}

In order to simulate the designs and observe energy absorption patterns, the tool used was COMSOL. It is a multiphysics simulation software with a finite element base. This software allowed us to design and compute several models and better understand the implications of all the properties of electromagnetic heating in the microwave spectrum. The package within comsol used was the RF (radio frequency) module, more specifically the microwave heating module. As said by one of the applications engineers at comsol, "Microwave heating is used when electromagnetic wave's wavelength is comparative to the device size". (Chris Pinciuc)

During the process of curing in a real case, the substrate changes significantly. The properties of the material change together with degree of cure, depending on the amount of cross linking the polymer has undergone. The rate of heat dissipation to the surroundings also affects the internal temperature and in turn the cure rate. Since the chip consists of several components only a part of which is the underfill and encapsulant that 
are to be cured, it would be difficult to simulate an exact version of an integrated circuit with the encapsulant and underfill. Studies show that due to the relatively small mass of these, the temperatures reached are significantly dependant on the properties of the adjacent chip and substrate. (Bailey, 2002)

These details and variations in them were not investigated in the scope of this project. In this study we attempt to understand the relationship of positioning and orientation of an FR4 substrate within a waveguide to its temperature change. It serves as a guide on two of the variables in this complex problem.

The software solves the general wave equation in the form of:

$$
\nabla \times\left(\mu_{r}^{-1} \nabla \times E\right)-k_{0}^{2}\left(\varepsilon_{r}-\frac{j \sigma}{\omega \varepsilon_{0}}\right) E=0
$$

Where $E$ is the electric field.

\section{$\underline{\text { Scalar inputs: }}$}

$k_{0}$ is the wave vector in free space

$\omega$ is the excitation frequency

$\varepsilon_{0}$ is vacuum permittivity

Domain inputs:

$\mu_{r}$ is the relative permiability 
$\varepsilon_{r}$ is the relative permittivity

$\sigma$ is the electric conductivity

The electromagnetic losses are modeled by the equations:

$$
\begin{gathered}
Q_{\text {electric }}=\frac{1}{2} \operatorname{Re}\left(\sigma E \cdot E^{*}-j \omega \varepsilon E \cdot E^{*}\right) \\
Q_{\text {magnetic }}=\frac{1}{2} \operatorname{Re}\left(-\frac{j}{\omega} \mu^{-1}(\nabla \times E) \cdot\left(\nabla \times E^{*}\right)\right)
\end{gathered}
$$

And the heat transfer into the substrate is calculated by:

$$
\rho C_{p} \frac{\partial T}{\partial t}+\nabla \cdot(-k \nabla T)=Q
$$

These equations are solved for tetrahedral free meshing elements with 4 nodes using a finite difference time domain (FDTD) formulation. This formulation allows for the calculation of the field intensities within the cavity and then using the acquired vectors to calculate energy loss. (D. Salvi, 2010) Tetrahedral elements are defined so as to have no other nodes than the vertices, and therefore the vector degrees of freedom do not have assigned position other than their association with edges or faces, and the scalar ones intermediate to the vertices. (Peter P. Silvester, 1996) 


\section{Closed cavity}

Most waveguides consist of a metal enclosure containing a transportation medium, usually air, for the microwaves. A closed cavity is used so as to prevent any loss by leakage and concentrate the microwaves.

In order to create an ideal scenario, the design consisted of a waveguide which itself served as the cavity in which the sample to be heated could be introduced.

To ensure the propagation of only a single mode, The lowest harmonic mode of TE10 was chosen. To attain this with a fixed frequency emitter, we can design the waveguide to have a cutoff frequency slightly below $2.45 \mathrm{GHz}$.

Using the equation for the cutoff frequency in TE 10 mode

$$
f_{c_{10}}=\frac{c_{m}}{2 a}<2.45 \times 10^{9} \mathrm{~Hz}
$$

In an air filled cavity, this implies:

$$
a>0.061 m
$$

In order to prevent higher modes of transmission, we calculate an upper limit of the length (a) so as to prevent TE20 transmission

$$
f_{c_{20}}=\frac{c_{m}}{a}>2.45 \times 10^{9} \mathrm{~Hz}
$$


Implying

$$
a<0.122 m
$$

The limitations that this implies that $0.061 m<a<0.122 m$

$$
\text { and } b<0.061 m
$$

To simplify the selection process, we can choose a standard waveguide and get dimensions of

\begin{tabular}{|l|l|l|l|l|}
\hline D band & WR-340 & 2.20 to $3.30(\mathrm{GHz})$ & $3.400 \times 1.700(\mathrm{in})$ & $86.36 \times 43.18(\mathrm{~mm})$ \\
\hline
\end{tabular}

(Rectangular waveguide Dimensions)

The standard waveguide is within the calculated extremes to allow only TE10 mode to propagate as $61.22 \mathrm{~mm}<\mathrm{a}<122 \mathrm{~mm}$ is the restriction.

$$
\lambda_{g}=17.34 \mathrm{~cm}
$$

\section{Changing size of the cavity}

Consumer microwaves also use this standard waveguide in order to tailor the output of the magnetron into TE10 mode.

Waves moving from a TE10 mode of propagation continue to propagate in the same mode even after the cavity size is changed after its initial alignment into TE10 mode.

The standing wave dimensions are the same in the larger cavity as those in the waveguide for cavity sizes that are multiples of the waveguides longer edge, and half the guide length, in their respective directions. 
An extension in the $x-y$ plane must be in multiples of the standing wave size, whereas extensions in the $\mathrm{z}$ direction can be freely done as the waves do not oscillate in this direction.

We know that the standing wave dimensions are the waveguide's $\mathrm{x}$ dimension (3.4 in), by $\lambda_{\mathrm{g}} / 2$.

If the cavity size is a multiple of these dimensions, a regular, easy to predict wave pattern is formed. Below are a few examples demonstrating this.

Time $=60$ Slice: Electric field norm $(\mathrm{V} / \mathrm{m})$

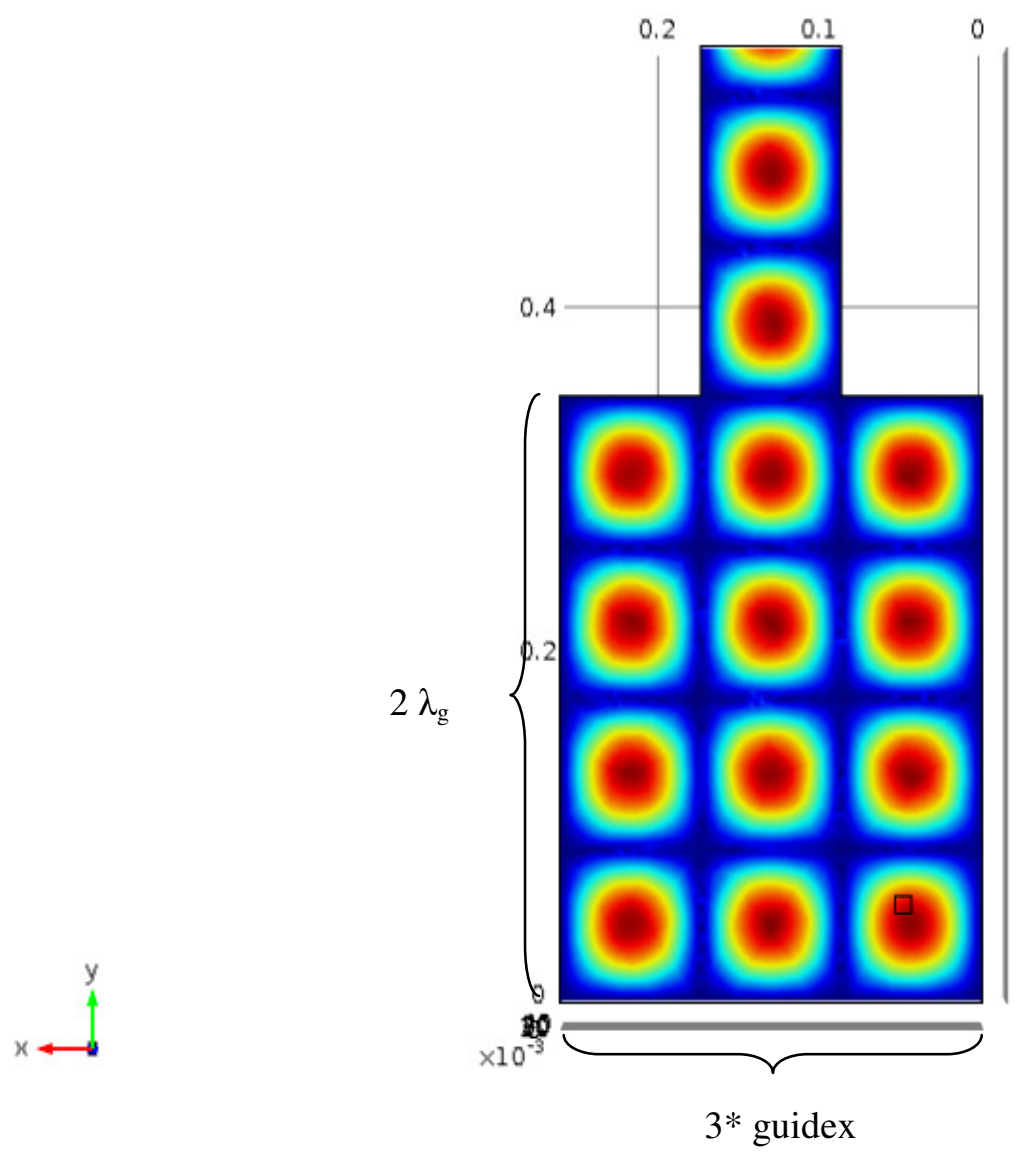

$\Delta 1.5397 \times 10^{4}$ $\times 10^{4}$

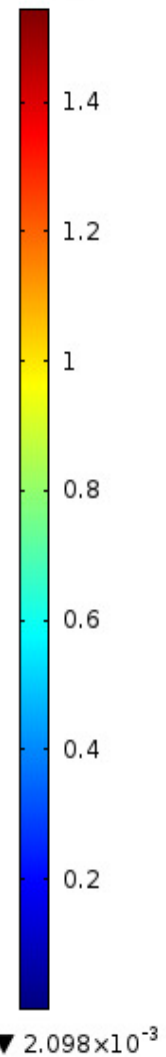

Figure 11: Electric field pattern in a regular sized cavity 
So long as the waveguide source is placed in alignment with the pattern that would be formed, this repetition of the pattern continues. A further increase of the cavity width in this multiple can be seen below.
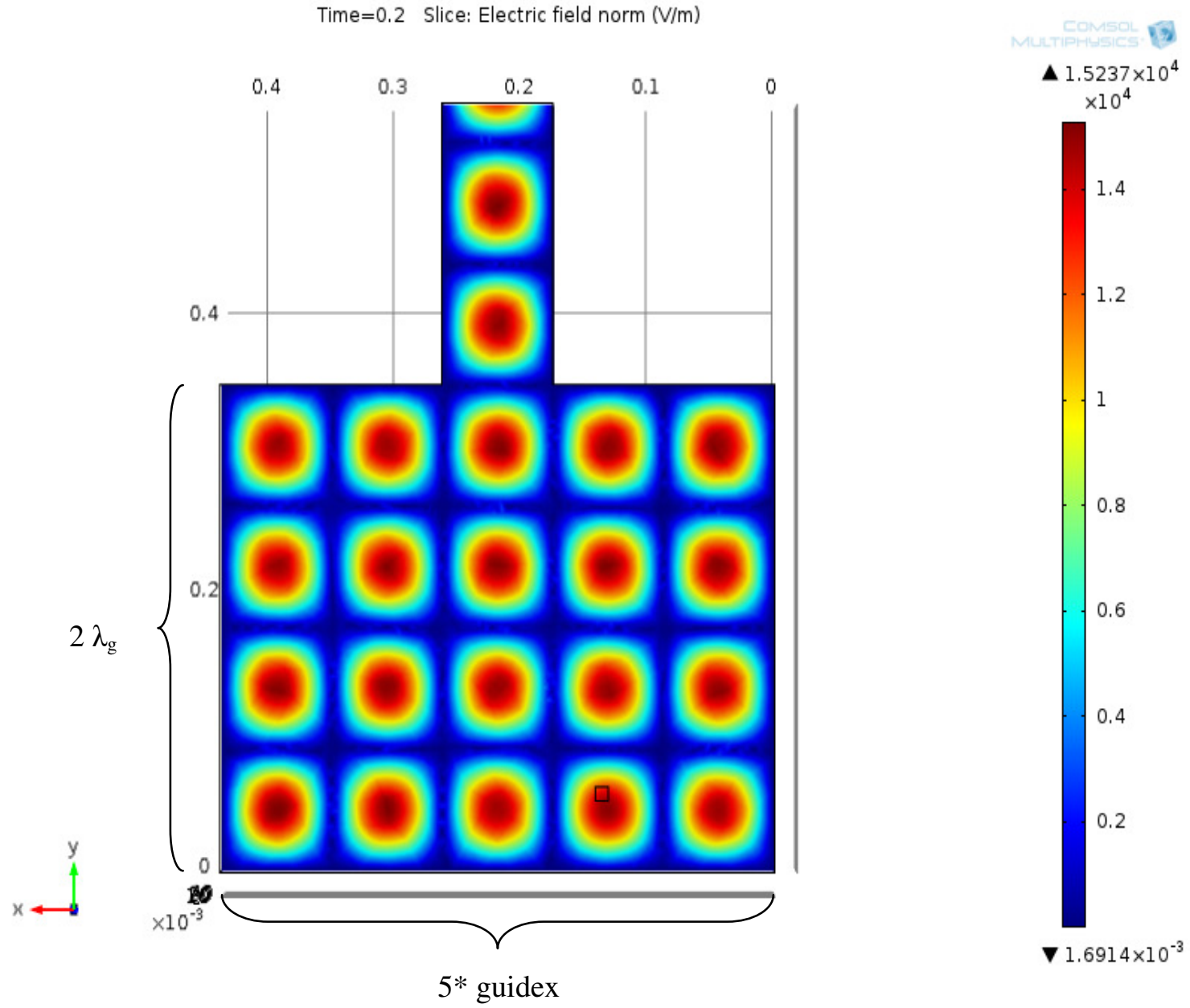

Figure 12:Field pattern when width is increased

After confirming the pattern's ideal repetition after an increase in the width, an increase in the length was tested for. As can be seen below, the same pattern continues. 

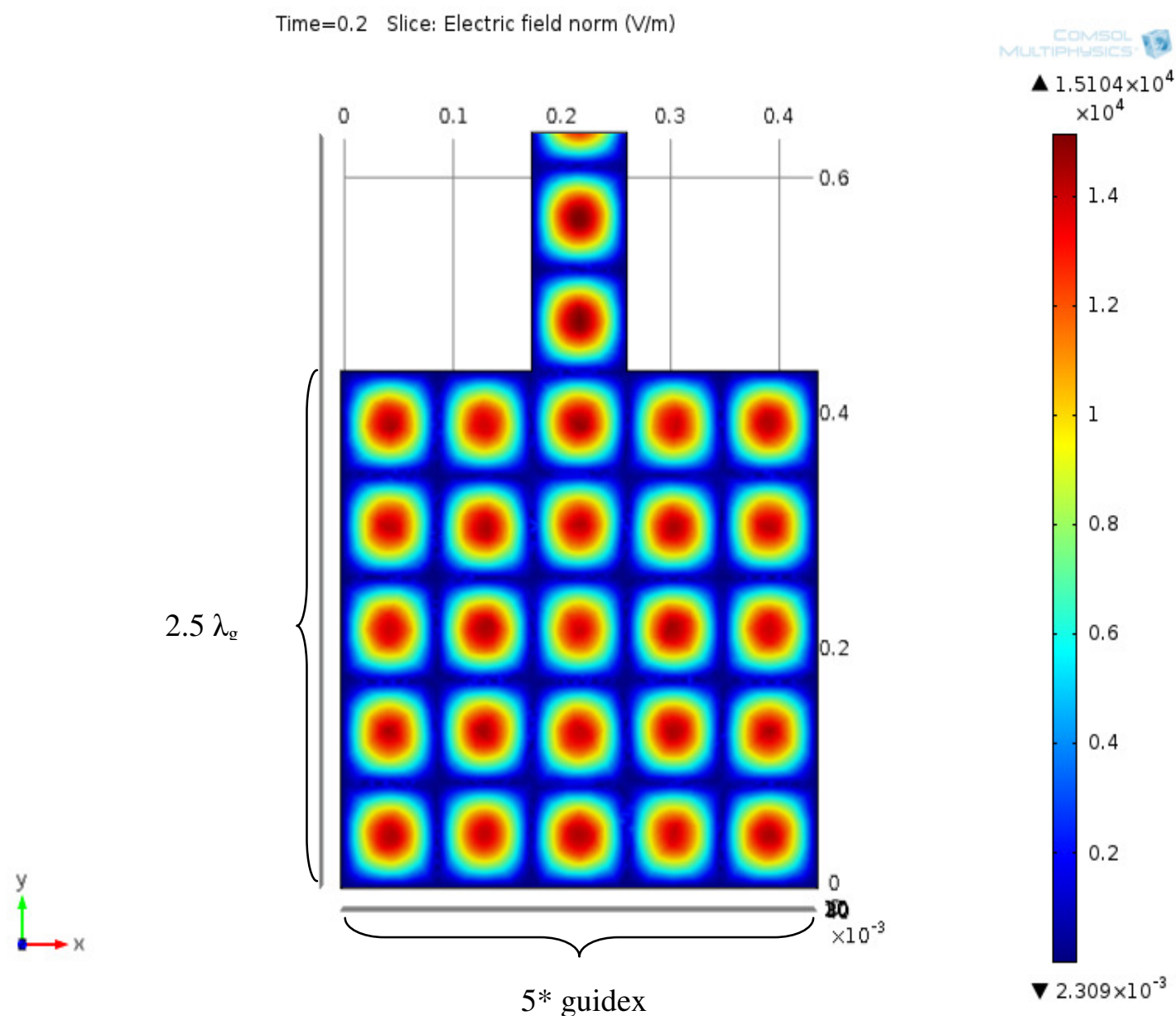

Figure 13: Field pattern when height is increased

By aligning the walls of the cavity and waveguide such that they always fall on the nodes of a prefect standing wave, such patterns can be obtained.

These perfect dimensions however have tight tolerances. Changing the size in the $\mathrm{x}$ and $\mathrm{y}$ dimensions even slightly, leads to a standing wave pattern that is difficult to calculate and predict. This can be seen below where the height of the cavity was changed by a quarter of the guide-wavelength. 
Time $=0.2$ Slice: Electric field norm $(V / m)$

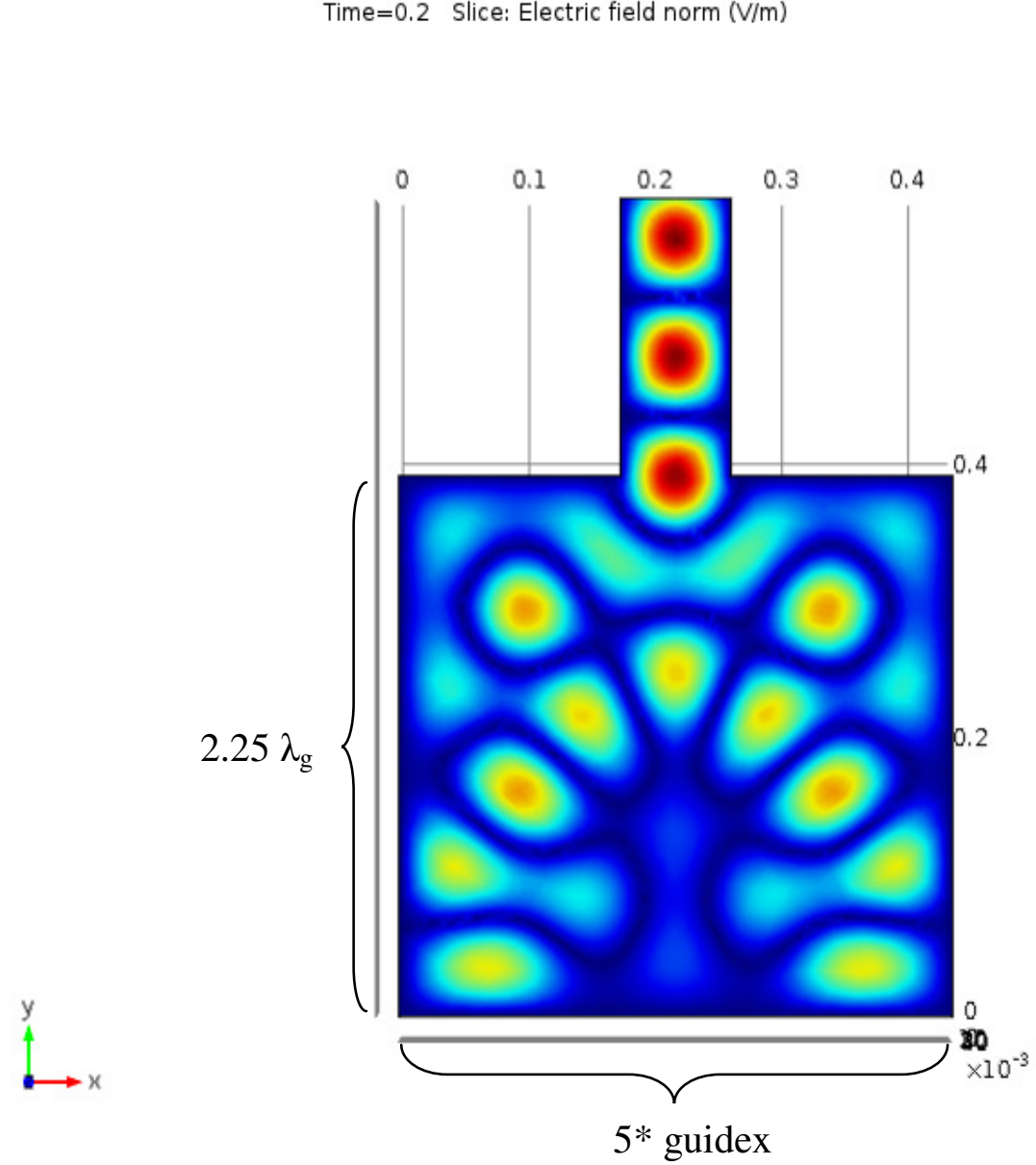

$\Delta 1.5371 \times 10^{4}$

$\times 10^{4}$

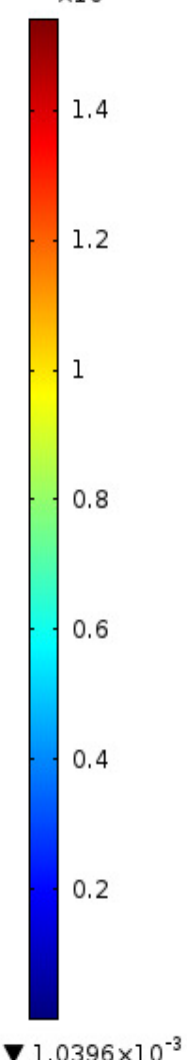

Figure 14: Field pattern for irregular geometry

This is the pattern with the height changed to $2.25 \lambda_{\mathrm{g}}$ and the width still being $5^{*}$ guidex.

The multiphysics solver in this case solves the general wave equation for a TE10 mode for the cavity with a central source as shown.

Though this gives hotspots divided by approximately the same distance as seen earlier in the ideal case, the position and intensity of them varies greatly.

This random pattern also confirms the results we had obtained earlier using the temperature distribution inside a domestic microwave cavity wherein hotspots were about 
2 columns, or about $10 \mathrm{~cm}$ apart. Here we get a result in the same ballpark of about $8 \mathrm{~cm}$, and given the roughness of the grid size of the measurement device in our physical model, this is a close result.

In the above calculations, we have only changed the planar $\mathrm{x}$ and $\mathrm{y}$ dimensions of the cavity, keeping the $\mathrm{z}$ dimension the same as the waveguide itself.

Changing the $\mathrm{z}$ dimensions of the cavity, we can see why conclusions could be made with the previous data alone.

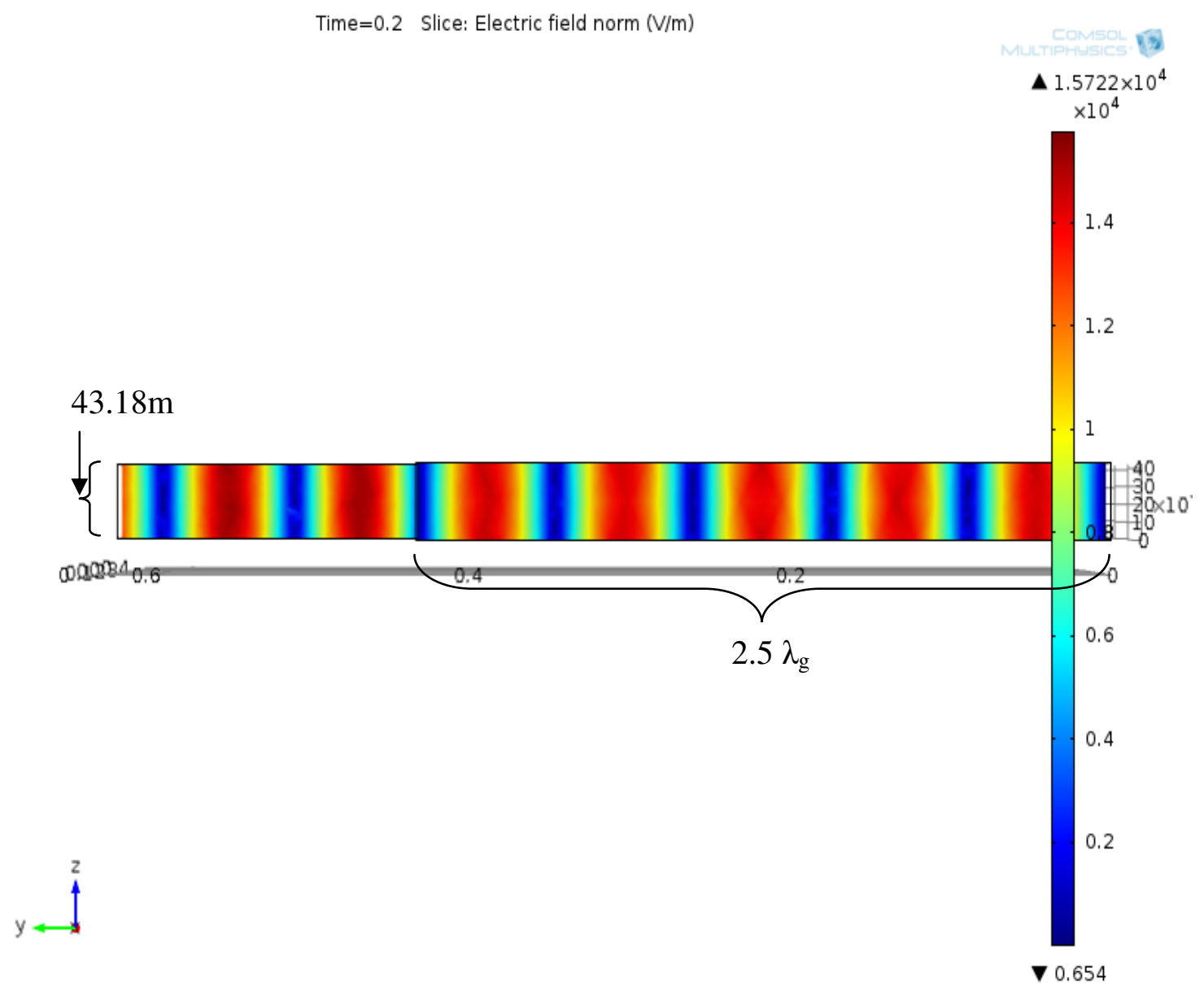

Figure 15: Field as seen along the smaller(vertical) direction [Along 0 resonance direction] 
Looking at the currently seen cavity from the side, we can see that the field does not vary with the cross-sectional position along the z-axis. Changing the cavity size, we can see the same happening for different size manipulations below.

Time $=0.2$ Slice: Electric field norm $(V / m)$

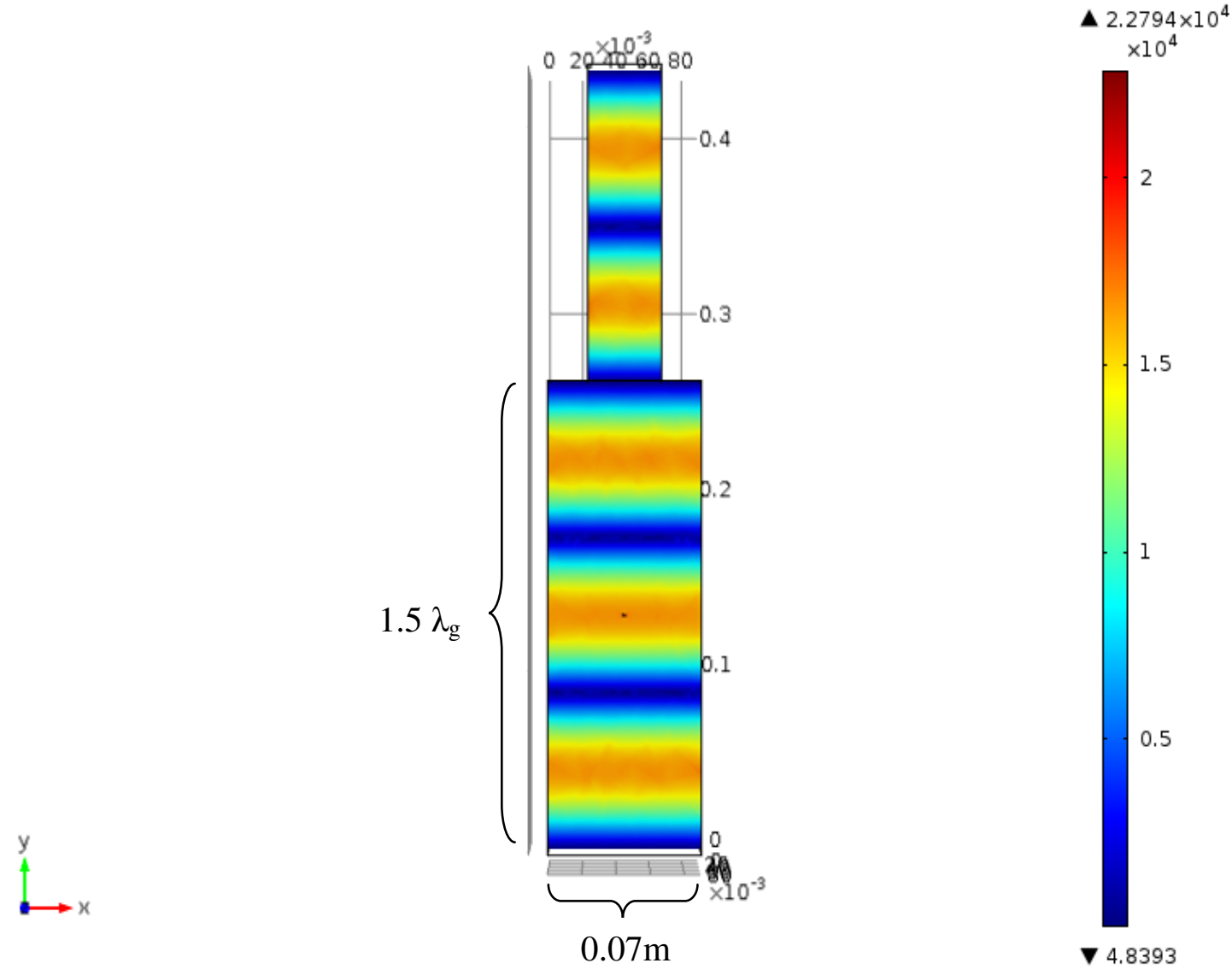

Figure 16: Field pattern after increasing height

Here we have a vertical size of $1.5 \lambda_{\mathrm{g}}$ by a horizontal dimension of $0.07 \mathrm{~m}$, which is greater than the waveguides size of $0.043 \mathrm{~m}$ in the same direction, we see that the mode of propagation does not change, and the field across the vertical direction is still the same.

To test the case of further propagation modes, we can increase this to a height that would allow TE11 propagation. 
Time $=0.2$ Slice: Electric field norm $(V / m)$

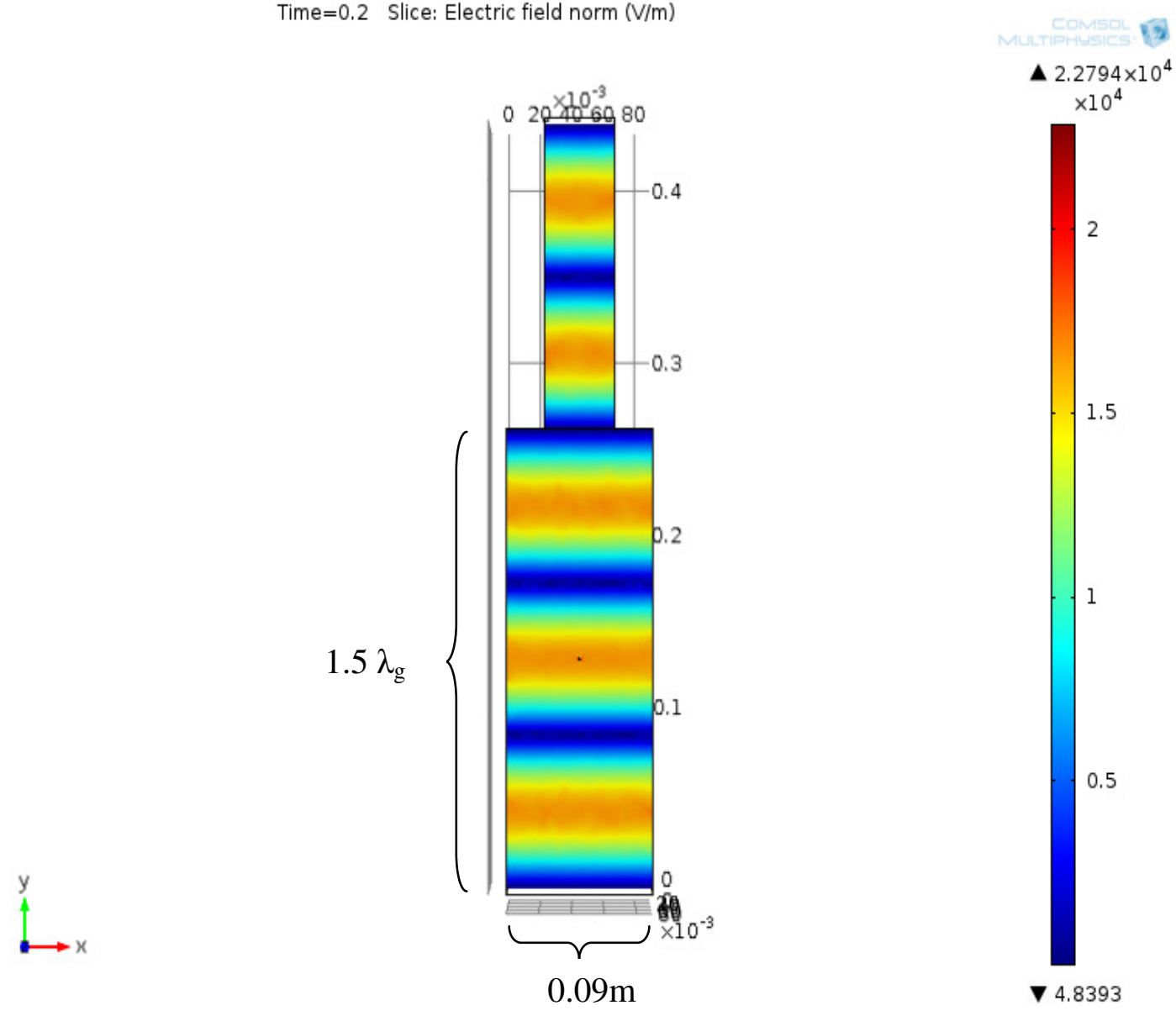

Figure 17: Field pattern when height increased further

Here the width is $0.09 \mathrm{~m}$, which should allow propagation of a second mode if the excitation had started here. As can be seen, the source alignment of propagation stays even though there is a change in the cavity size. 


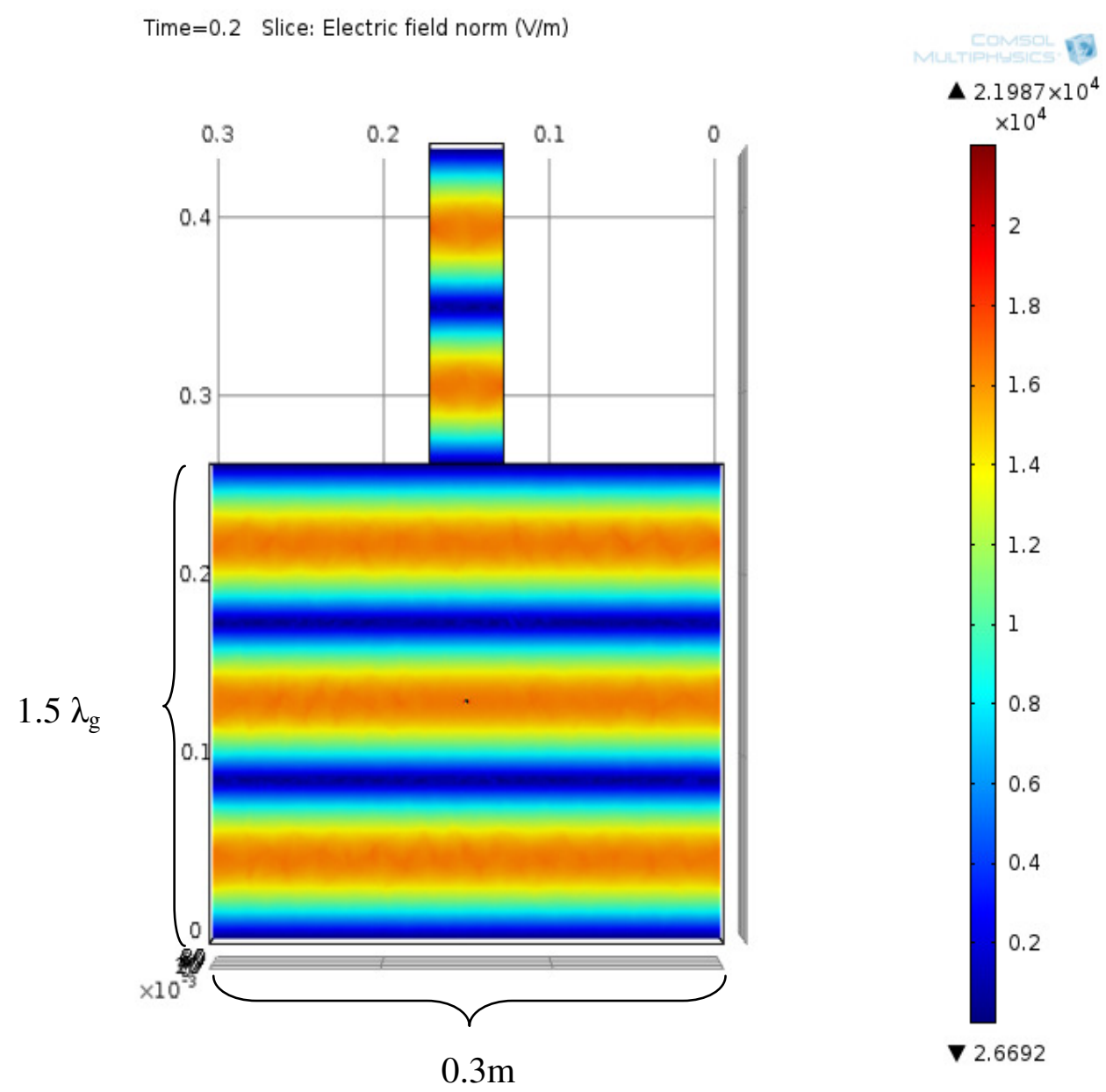

Figure 18: Field pattern after significant increase in height

Changing the dimensions of the cavity side along the 0 propagation mode direction to $30 \mathrm{~cm}$ still does not affect the standing wave pattern.

Changing the z-dimension of the cavity can therefore be concluded to have no effect on the resultant standing wave pattern, and therefore decisions made in cavity design can be based on the planar $\mathrm{x}$ and $\mathrm{y}$ dimensions alone. 
Gaining a basic understanding for the energy distribution inside the microwave cavity, we can use this information in order to position and place the substrates in the cavity with good sense.

In order to test the designs, the substrate used to measure the power absorbed is an FR4 block of dimensions $40 \mathrm{~mm} \times 40 \mathrm{~mm} \times 1 \mathrm{~mm}$. This is larger than an average sized BGA chip which has dimensions $23 \mathrm{~mm}$ per side with a height of about $1 \mathrm{~mm}$. (Flip Chip Ball Grid Array Package Reference Guide, 2005) thus allowing a large margin for variability in chip design.

The power output used in the simulations is $120 \mathrm{~W}$. This is in order to reflect the physical model that was constructed, operating at $10 \%$ output of a $1200 \mathrm{~W}$ source. 


\section{Model}

We start our investigation into the heating of a substrate by comparing the effect of reorienting the substrate in a cavity with only TE10 mode propagating.

The samples to be heated using these microwaves must mimic real world underfills used. For this reason, the sample chosen was FR4. FR4 has a value of thermal conductivity of $0.3[\mathrm{~W} / \mathrm{mK}]$, this is very similar to epoxies used in underfill which have the same constant measuring 0.23[W/mK]. (Woong Sun Lee, 2005)These adhesives are constantly studied so as to improve the thermal conductivity using fillers and the like. The current results surpass the thermal conductivity of FR4 in many cases. FR4 therefore provides a good ballpark result of how these substrates would behave.

The model was defined as a cavity with the dimensions of a standard waveguide (WR340) with one side that operated as the source and sink of the microwaves into the cavity. This plane has the rectangular dimensions of the standard waveguide in order to ensure propagation of only TE 10 mode in a direction normal to the plane. 


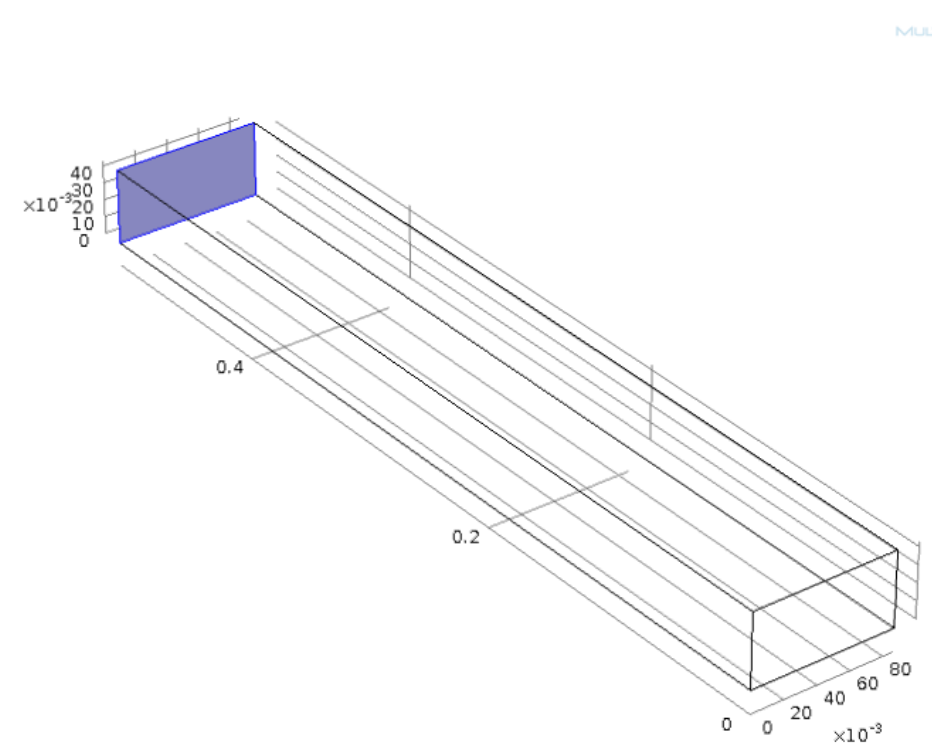

Figure 19: Model of standard waveguide with highlighted source

All other surfaces were defined as perfect conductors so as to serve as surfaces to reflect the waves.

With the TE10 mode of propagation within the waveguide, the square chip is aligned so as to make the centre of the chip co-inside with the maximum in the standing wave produced. This position is acquired by knowing the fact that the end surface will be a nodal surface and therefore there will be a hotspot or a maxima every $\frac{1}{4} \lambda_{g}+n \frac{1}{2} \lambda_{g}$ from the nodal surface. In this case the chip was placed with its centre at $1 \frac{1}{4} \lambda_{g}$ from the far end of the cavity. This allows us to observe the best case scenario for a chip in a microwave cavity as the hotspot is where the field intensity of the microwaves is maximized. 
Position 1: Horizontal chip with centre of chip in centre of hotspot

In the first case, the smaller dimension of the chip is aligned along the $\mathrm{z}$-axis while the wave propagates in the y-axis. This arrangement was used trying to achieve the lowest temperature gradient within the chip.

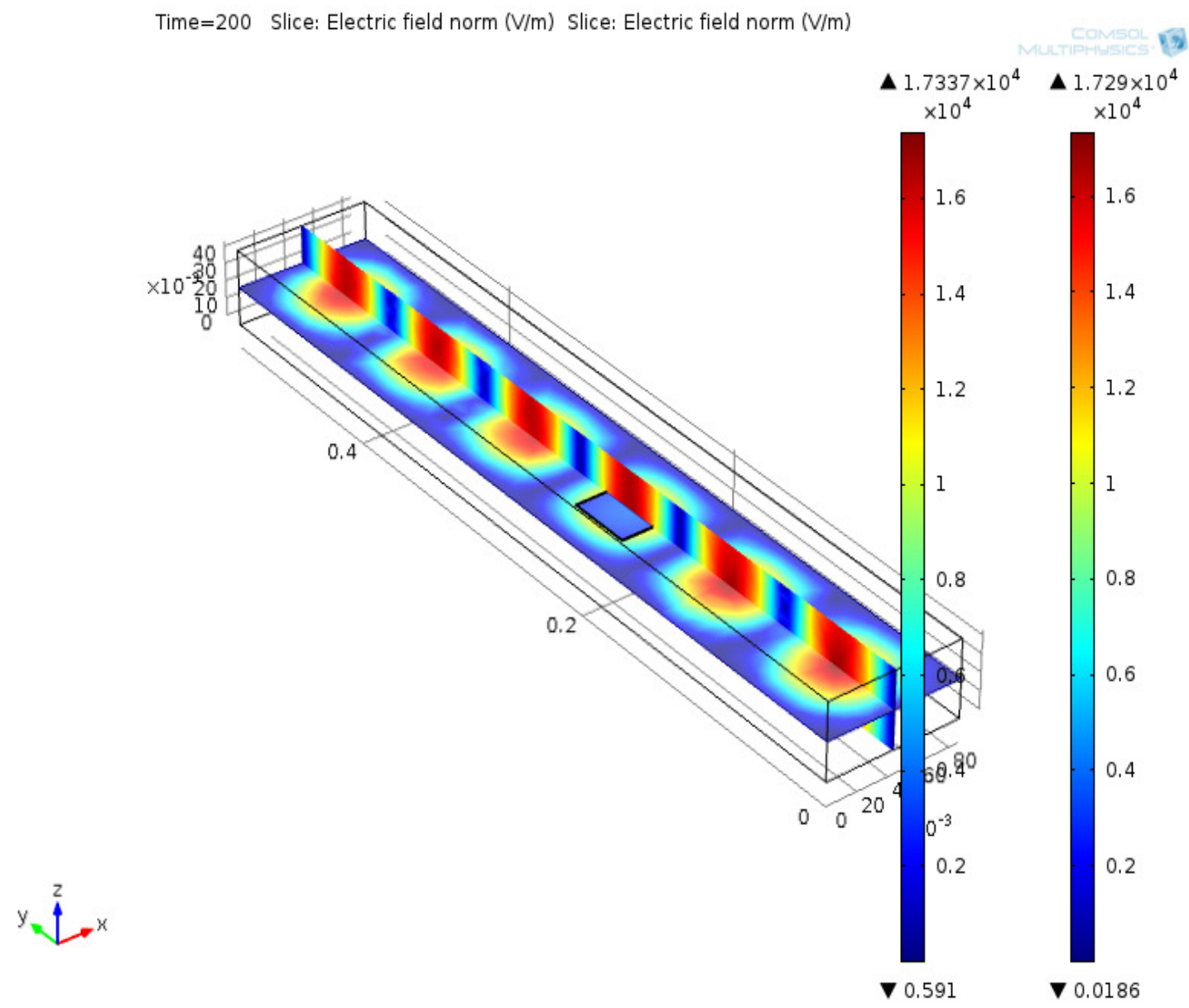

Figure 20: Field pattern and horizontal substrate placement inside waveguide

Looking at a horizontal cut of the entire cavity through the centre of the chip, we can observe the change in the field, and see how it exists inside and outside the substrate. 


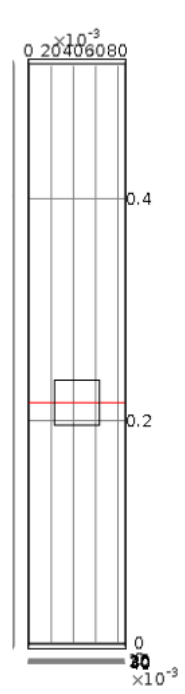

Figure 21: Cut line showing measurement points across the cavity

The cutline shown above goes from one end of the waveguide cavity to the other horizontally, allowing us to see the transition from one node, through the air cavity and the chip. We can observe the changing electric field through this below. 


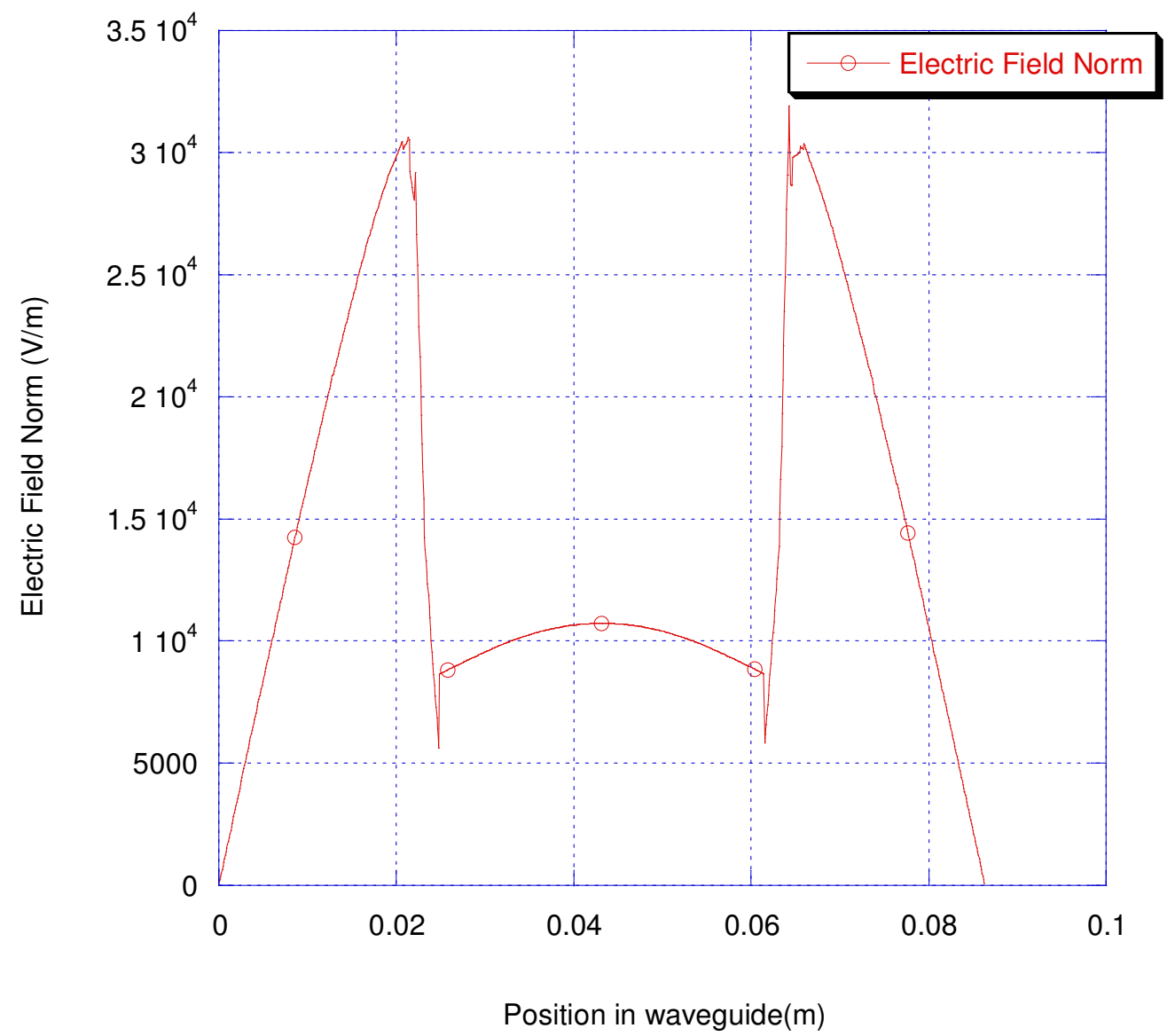

Figure 22: Electric field norm through the cavity in the presence of a horizontal substrate

As can be seen, the field drops sharply within the substrate due to the substrate having very different values from air for its electric permittivity $(\varepsilon)$.

Calculating these results numerically, we get a very similar trend. The discontinuities at the boundary of the chip and air are the only ones unaccounted for. These discontinuities 
are caused partly due to comsol's mesh size and also due to approximation errors in the calculations performed. The surface area of the chip being exposed to the electric field in air is also increased on the edges due to the extrusion of $1 \mathrm{~mm}$ in the vertical dimension. These minor discontinuities are therefore understood in this context.

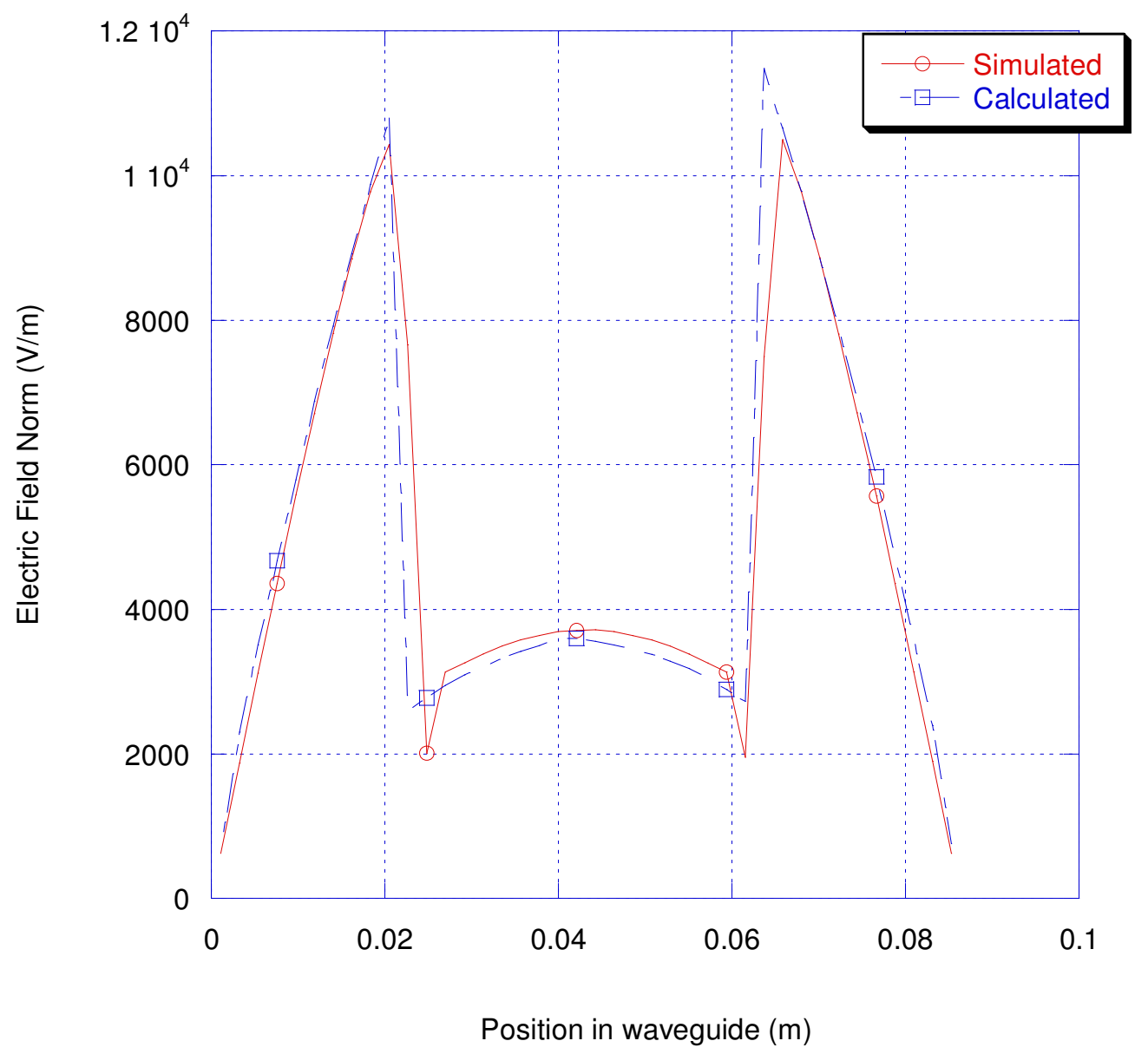

Figure 23:Electric field through the cavity in the presence of a horizontal substrate 
Observing only the chip and taking a horizontal cut as shown,

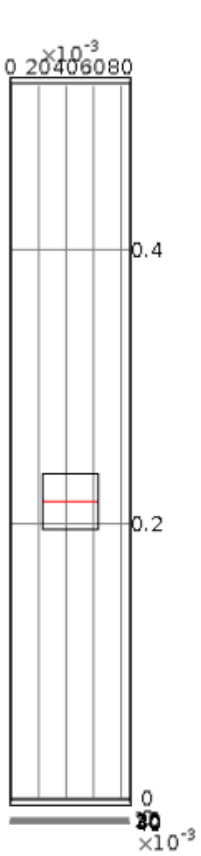

Figure 24: Cut line through only the chip as measurement points

We can look at the details of the electric field by zooming in into the chip. Below are the details for the electric field norm inside the chip. 


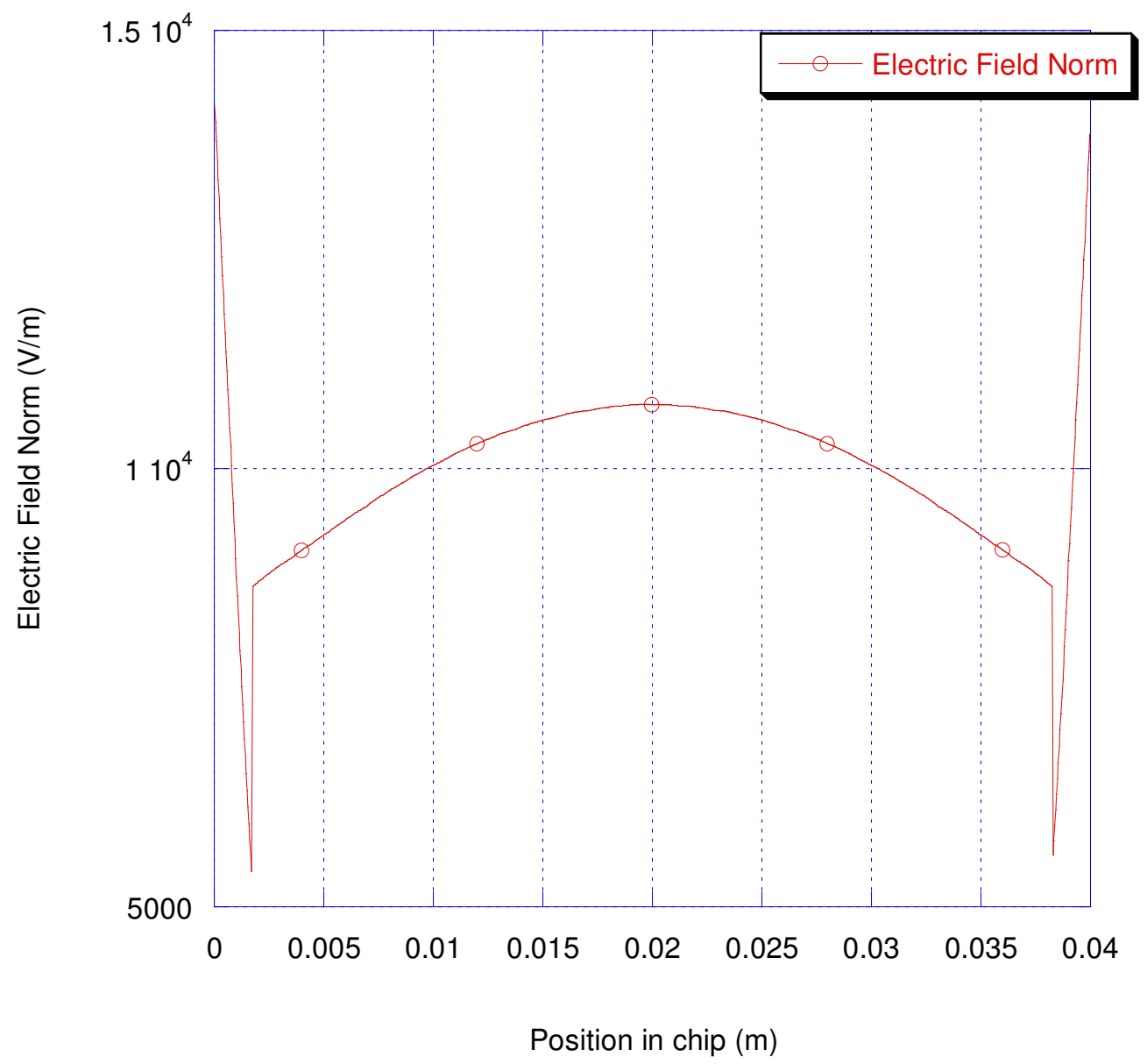

Figure 25: Electric field norm inside the horizontal chip

This electric field leads to a temperature change in the chip through the process of dielectric heating. The resultant temperature change profile is shown after. In order to observe a heating profile with a minimal effect of conduction, we can observe the temperature change after a very short time. In this case $0.1 \mathrm{~s}$ is used. Comparing the results of the simulation with the calculated values, we get the observation below. (Wang, 2000) 


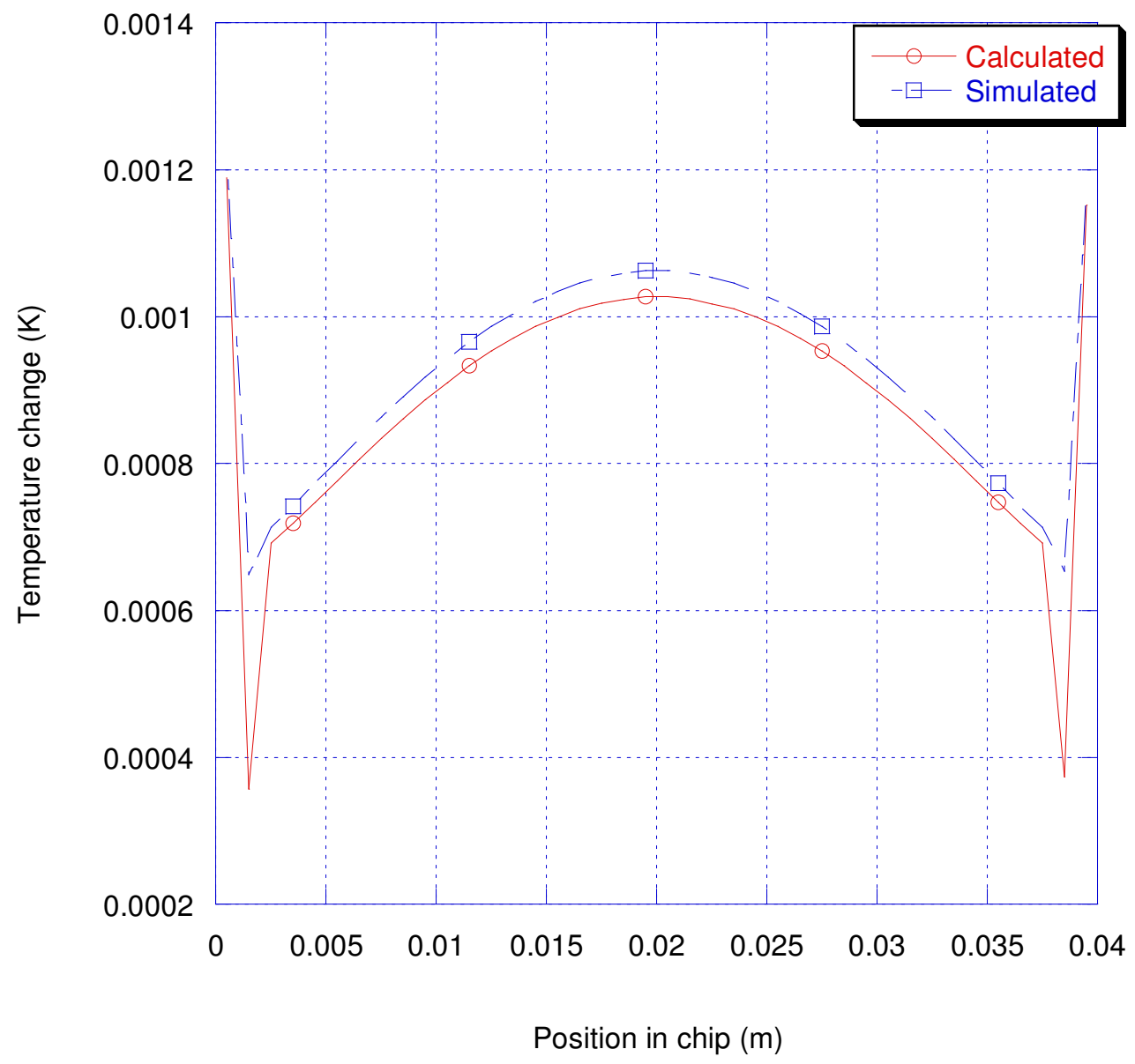

Figure 26: Temperature change in 0.1second of heating

The calculated values are slightly off from the simulated values due to the approximation assumed in the loss tangent of the material. This value is known to vary depending on frequency and temperature (Wang, 2000), the error is therefore more due to a different 
value being assumed by the software and in the analytical equations. Another source of this error is neglecting conduction at this short period of time in the analytical study.

This temperature profile changes with time due to conduction playing a more significant role at larger times. After 10 seconds of heating, the profile changes to that seen below.

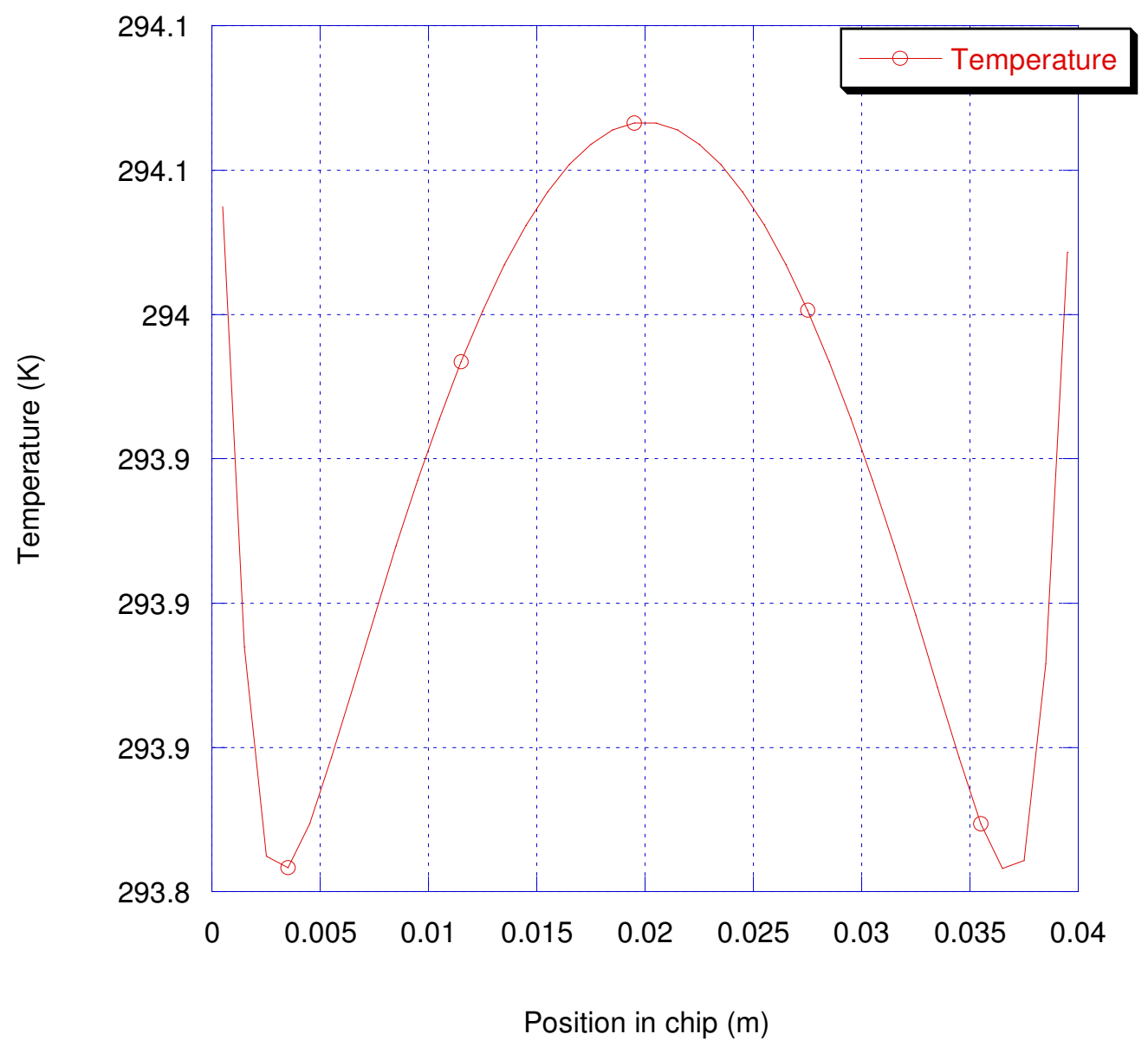

Figure 27: Temperature in the horizontal chip after 10 seconds of heating 
This graph differs from the previous in the fact that it accounts absolute temperature as opposed to temperature change. The effect of conduction can be seen clearly as the larger gradients of temperature are lowered.

After a certain amount of time has passed, the temperature profile is constant as the conductive effects and microwave heating effects balance. This can be seen below in a set of graphs where temperature is recorded at every 250 seconds. 


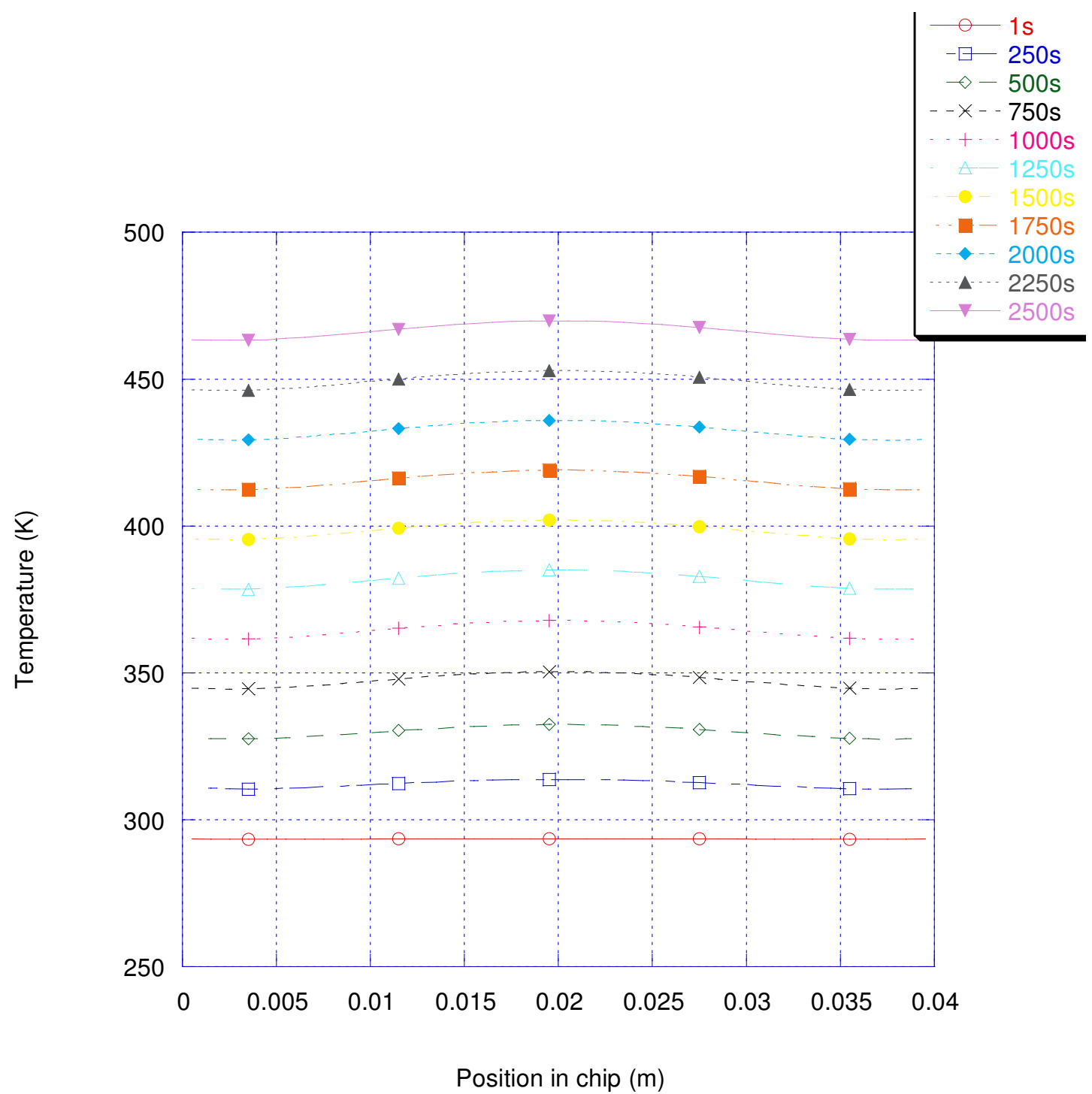

Figure 28: Temperature inside the chip at 250s intervals

Position 2: Vertical chip with centre of chip at the centre of a hotspot In this case, the chip is angled so that the smaller dimension of the chip is oriented along the y-axis, the same as the propagation direction of the waves in the cavity. 


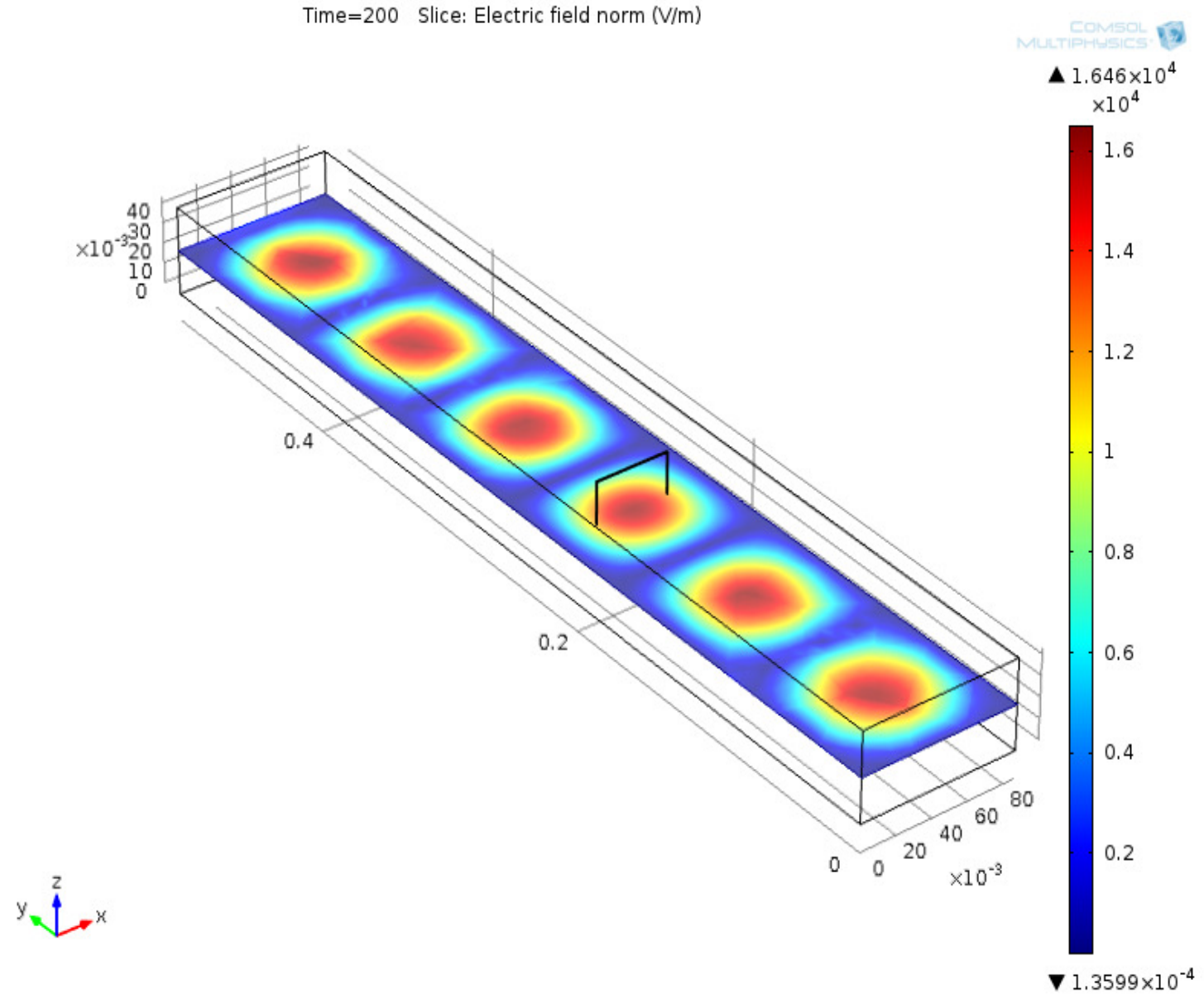

Figure 29: Field pattern and vertical substrate placement inside the waveguide

Due to the thickness being so small $(1 \mathrm{~mm})$ in the direction of propagation of the waves, almost no attenuation in the electric field occurs inside the chip as compared to the outside. 
Taking a horizontal cut of the entire cavity, we can see this occur.

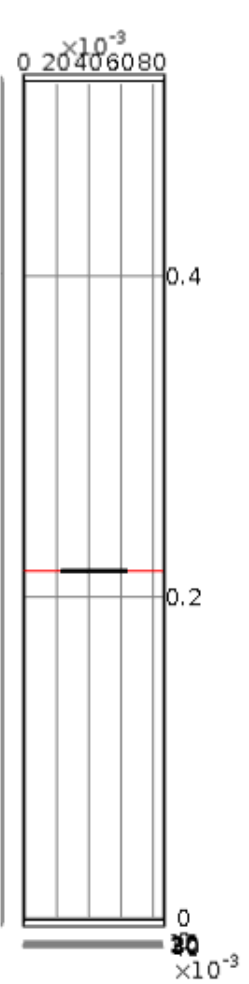

Figure 30: Cut line through the cavity to specify measurement points 


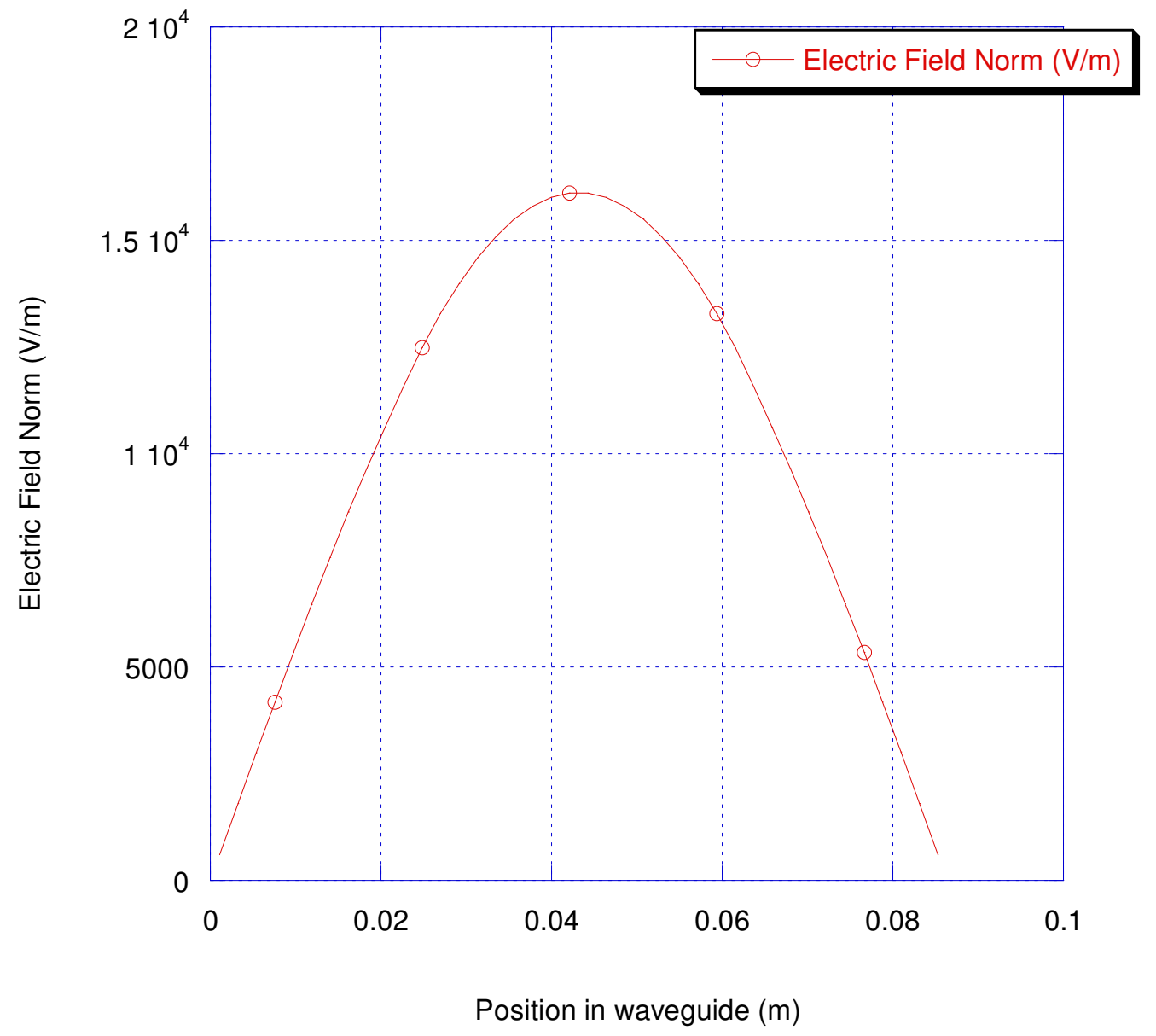

Figure 31: Electric field norm through the cavity in the presence of a vertical chip

This arises from the fact of the reflected waves within the waveguide not having to pass through a large distance within the FR4 before reaching this point (line). 
Focusing inside only the chip, we can see the electric field is significantly more than the chip in the horizontal orientation.

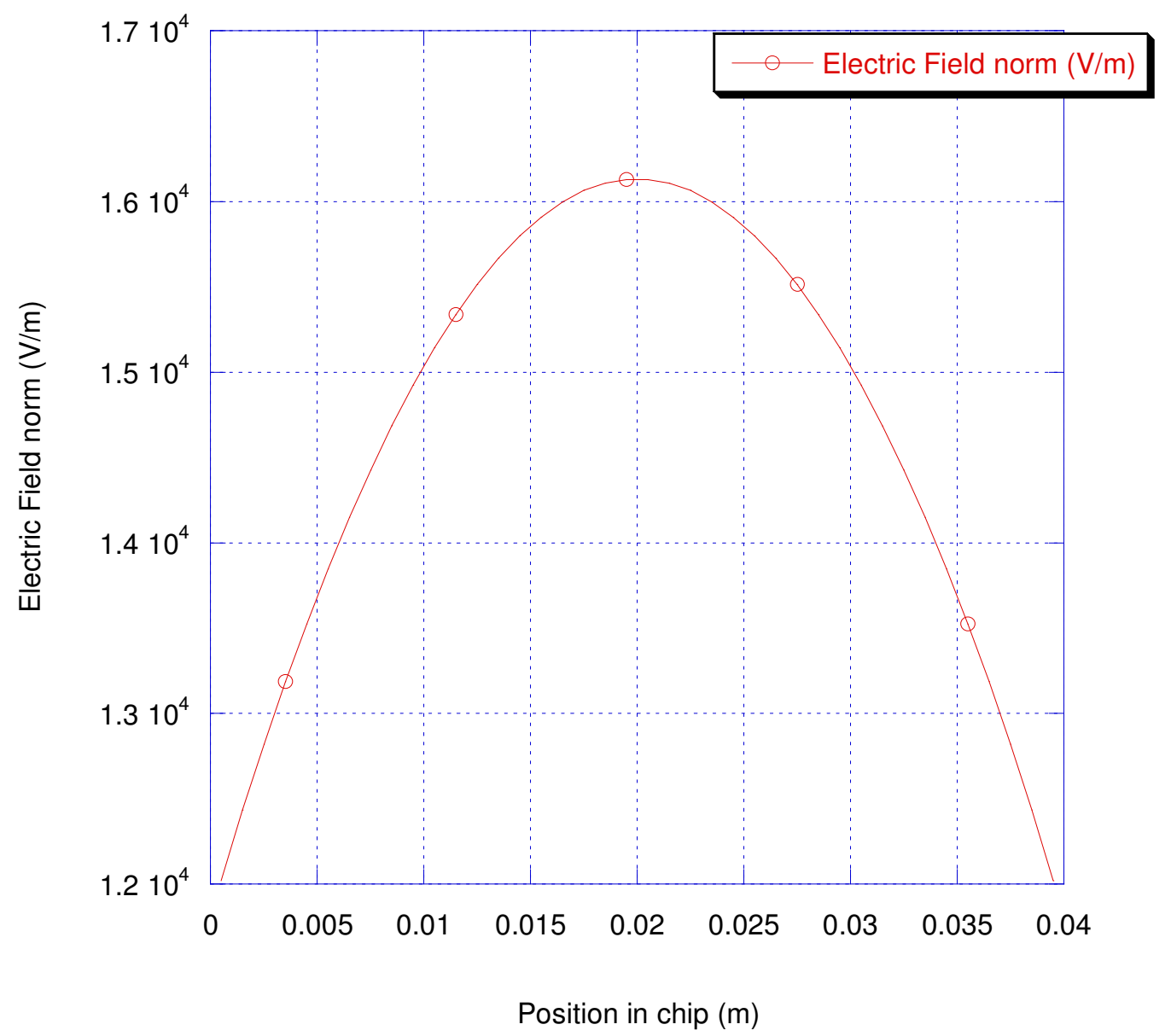

Figure 32: Electric field norm inside the chip placed vertically in the cavity

The electric field at the centre here is about $1.6 \times 10^{4} \mathrm{~V} / \mathrm{m}$ as compared to $1.1 \times 10^{4} \mathrm{~V} / \mathrm{m}$ in the horizontal orientation. 
This leads to a higher temperature with a profile as shown below after 10 seconds of heating.

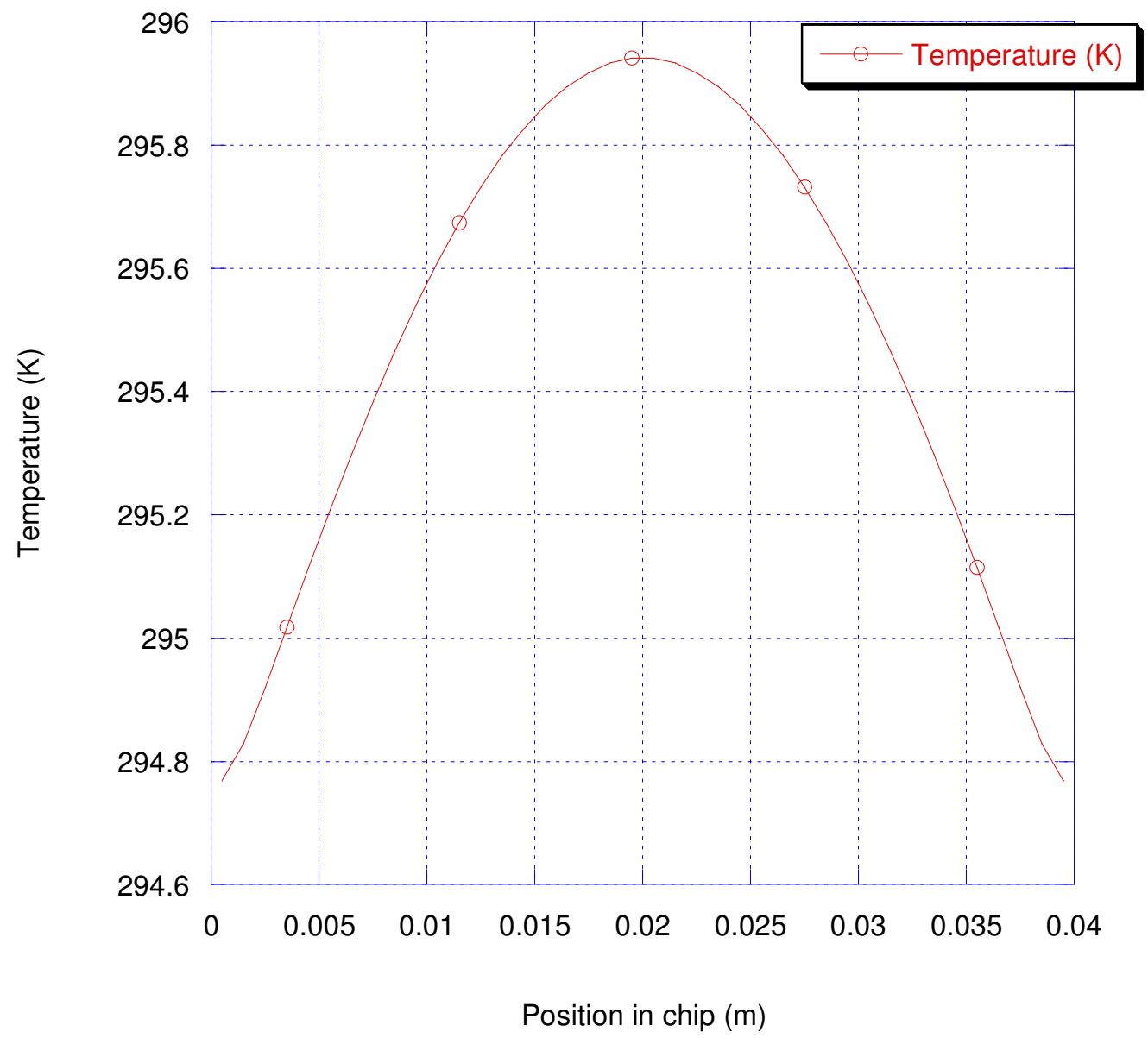

Figure 33:Temperature in the substrate after 10s of heating

This orientation of the chip leads to significantly higher temperatures inside the chip.

This is proportional to the square of the electric field inside the chip, and since the 
attenuation of the electric field is lower in this orientation, the increased heating up is justified.

After a significant amount of time undergoing microwave heating, the conduction within the chip together with the microwave heating normalizes the temperature profile to a constant. This can be seen below where the temperature profile is plotted every 250 seconds. 


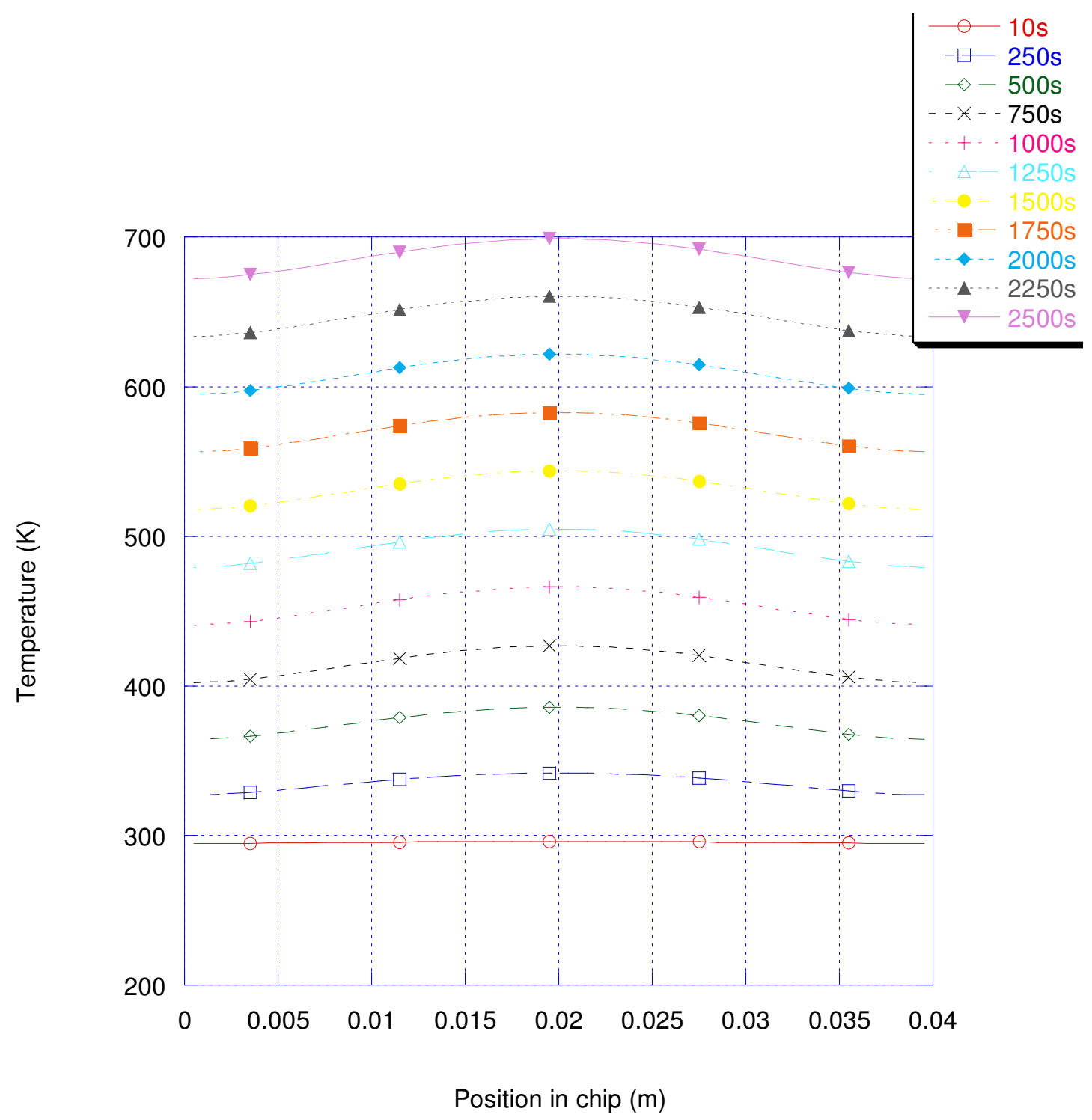

Figure 34:Temperature inside the chip at 250s intervals

The field attenuation increases with increasing thickness of the chip when in the vertical orientation. As can be seen below, 


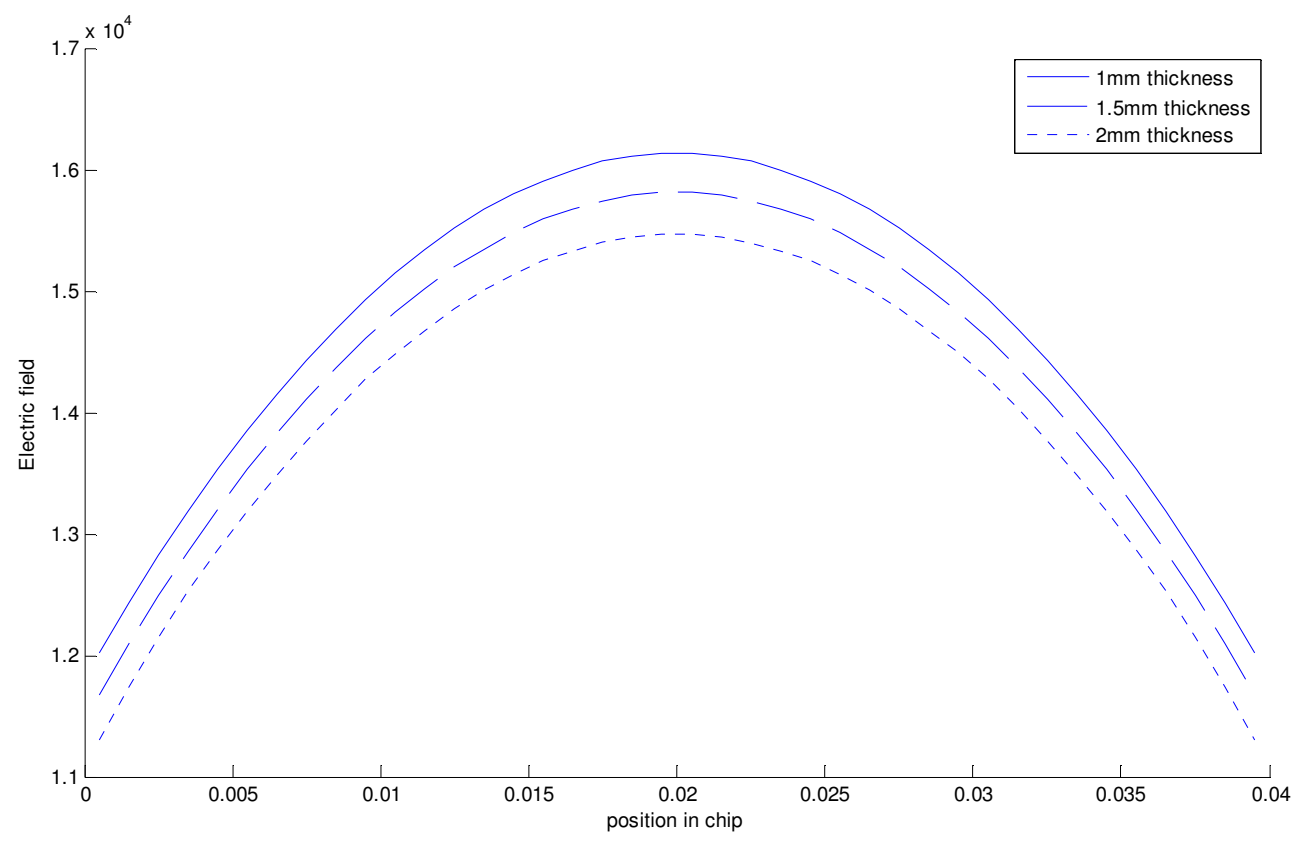

Figure 35: Electric field norm inside the vertical chip with changing thickness

When changing the thickness when the chip in this orientation, the field inside the chip decreases due to this increasing thickness change due to the increase being in a direction parallel to the propagating direction. The wave must mass through a larger portion of the substrate to reach this point.

The effect of a change in thickness in the horizontally placed chip can be seen below. The results are quite different. 


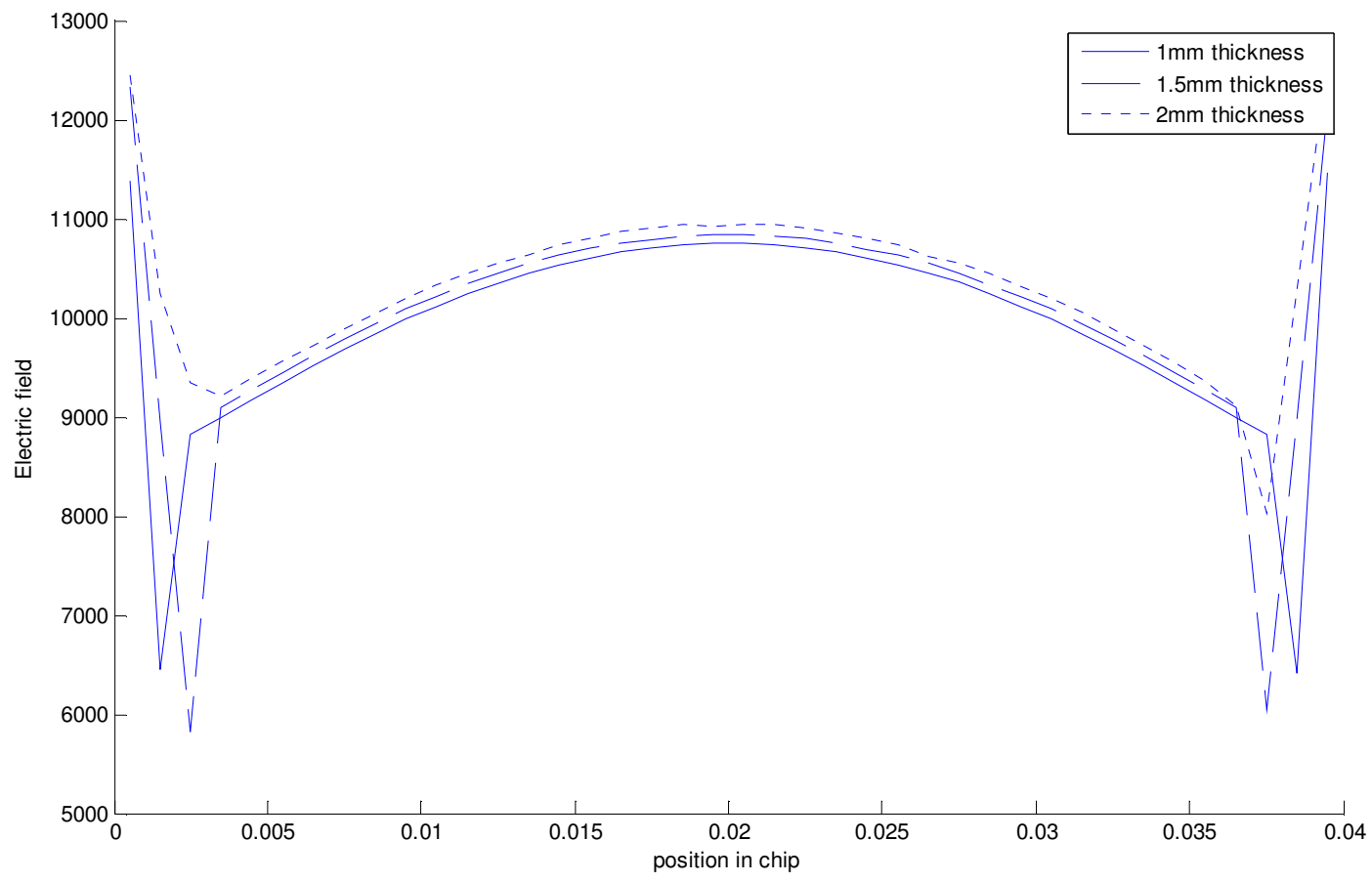

Figure 36: Electric field norm inside a horizontal chip with changing thickness

In the horizontally placed chip, the change in electric field inside the chip is minimal, and to an extent increases with increase in thickness. This is due to the thickness increasing in a direction perpendicular to the propagation direction of the wave front, and therefore having a larger surface perpendicular to the propagation direction, and thus allowing a larger absorption surface. 


\section{Open Cavity}

An open microwave is a waveguide with an open end that creates a useable evanescent field on the open end. It works on a principle of designing the waveguide such that the dimensions of the waveguide allow propagation of the electromagnetic wave given a certain medium( in this case PTFE) that has a higher dielectric constant, but do not allow for propagation if the waveguide had air as its internal medium of propagation. This change in the medium creates a barrier for the waves and they are internally reflected. The air filled part of the cavity however still has an evanescent electric field induced by the existence of an electric field in the other filler material, in this case PTFE.

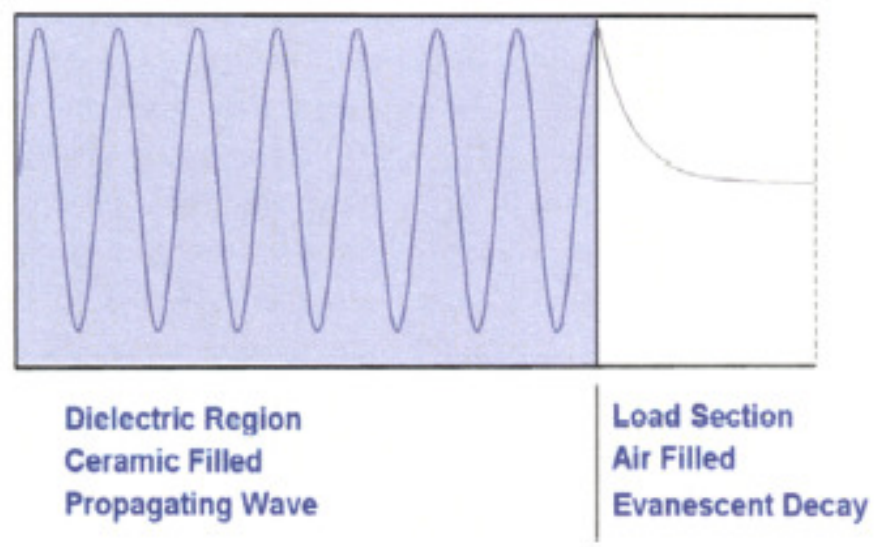

Figure 37: Open microwave cavity

(K.I. Sinclair, 2008)

\section{Design}

Looking at the works of Sinclair et al., open ended microwaves operate in the TM mode in order to maximize the evanescent field at the open end. The cavity for this mode however supports propagation of multiple other modes, starting from TE10 to the mode designed for (usually TM11). This implies the presence of the TE10, TE01, TE11 and 
TM11 modes. In order to simplify the application process, we have limited the design so as to allow only a single mode (TE10). This ensures a confirmed mode of propagation, and a known cutoff so as to manage no leakage in any propagation mode. (Sumanth Kumar Pavuluri, 2012)

Following by the example of (K.I. Sinclair, 2008), the open cavity was designed with a dielectric insert in the waveguide in order to maintain total internal reflection and prevent a propagating wave from exiting the cavity.

The Frequency Agile Microwave Bonding System(FAMOBS) developed by Sinclair et al. uses a variable frequency in a higher frequency range.(8-12GHz) (T. Tilford, 2007). This design has a very small output in a size comparison as the size of the standing wave produced is significantly smaller. It is being developed to be able to cure precision parts and substrates. The design uses the evanescent field emanating from the open end of the microwave cavity in order to provide energy to cure samples. These evanescent fields exist only for a short distance outside the waveguide and die out exponentially. This is an advantage in the safety aspect as leakages are prevented.

A schematic view of the waveguide from its side is shown below. The walls of the waveguide are made of metal, and inside, it consists of a PTFE section, followed by a Dielectric insert, which in this case is mica, followed by an air cavity that is open to the outside. It is here that the chip would ideally be placed. 


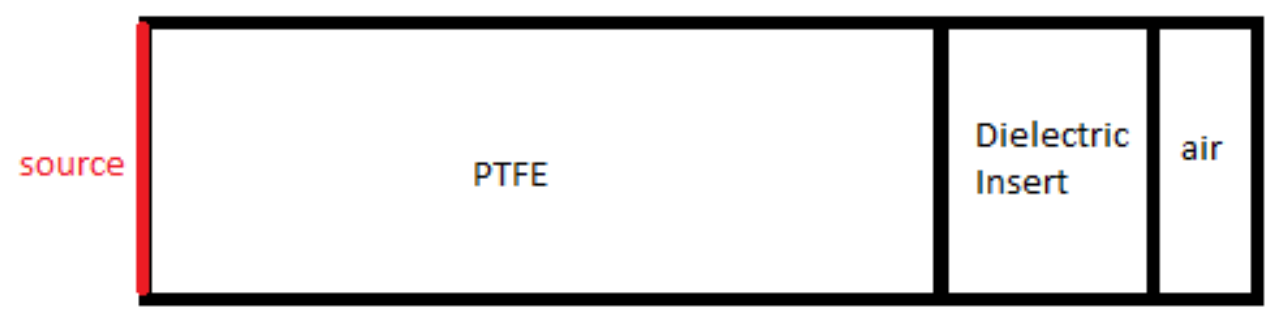

\section{Figure 38: Open air cavity design}

In order to ensure this field response, the waveguide must be designed so that the cutoff frequency of the PTFE and mica section is above the source frequency, and that of the air column is below the frequency used. This implies that the wave will propagate through the PTFE and dielectric insert, and then get reflected as it will not propagate through the air cavity. The air cavity will only have an evanescent electric field which will die out exponentially.

The PTFE and dielectric insert are materials selected due to their relatively high relative permittivity and low loss tangent. These material properties allow for the design dimensions to have the resultant of propagation in the dielectric materials but attenuation in the air cavity.

the PTFE and mica waveguide sections therefore must have allow only TE10 mode of $2.45 \mathrm{GHz}$ radiation to propagate.

PTFE material properties:

Relative permittivity $\left(\varepsilon_{r}\right)=2.1$ 
Relative permeability $\left(\mu_{r}\right)=1$

Mica material properties:

Relative permittivity $\left(\varepsilon_{r}\right)=5.8$

Relative permeability $\left(\mu_{r}\right)=1$

These properties lead to the result being

$$
0.042245 m<a<0.059743 m
$$

\section{$0.042245 m>b$}

using these results as guidelines, we define the cavity to have a cross section of dimensions $40 \times 55 \mathrm{~mm}$. This ensures the transmission of only TE10 mode within the cavity. These dimensions also ensure that no modes will propagate in the air cavity, thus confirming that only evanescent fields will exist in the air cavity.

The insert of a material with a higher dielectric constant is used (mica/Borosilicate glass) in order to maximize the evanescent field at the exit. To optimize the size of the insert, simulations were run varying its size and comparing the evanescent field thus formed. 


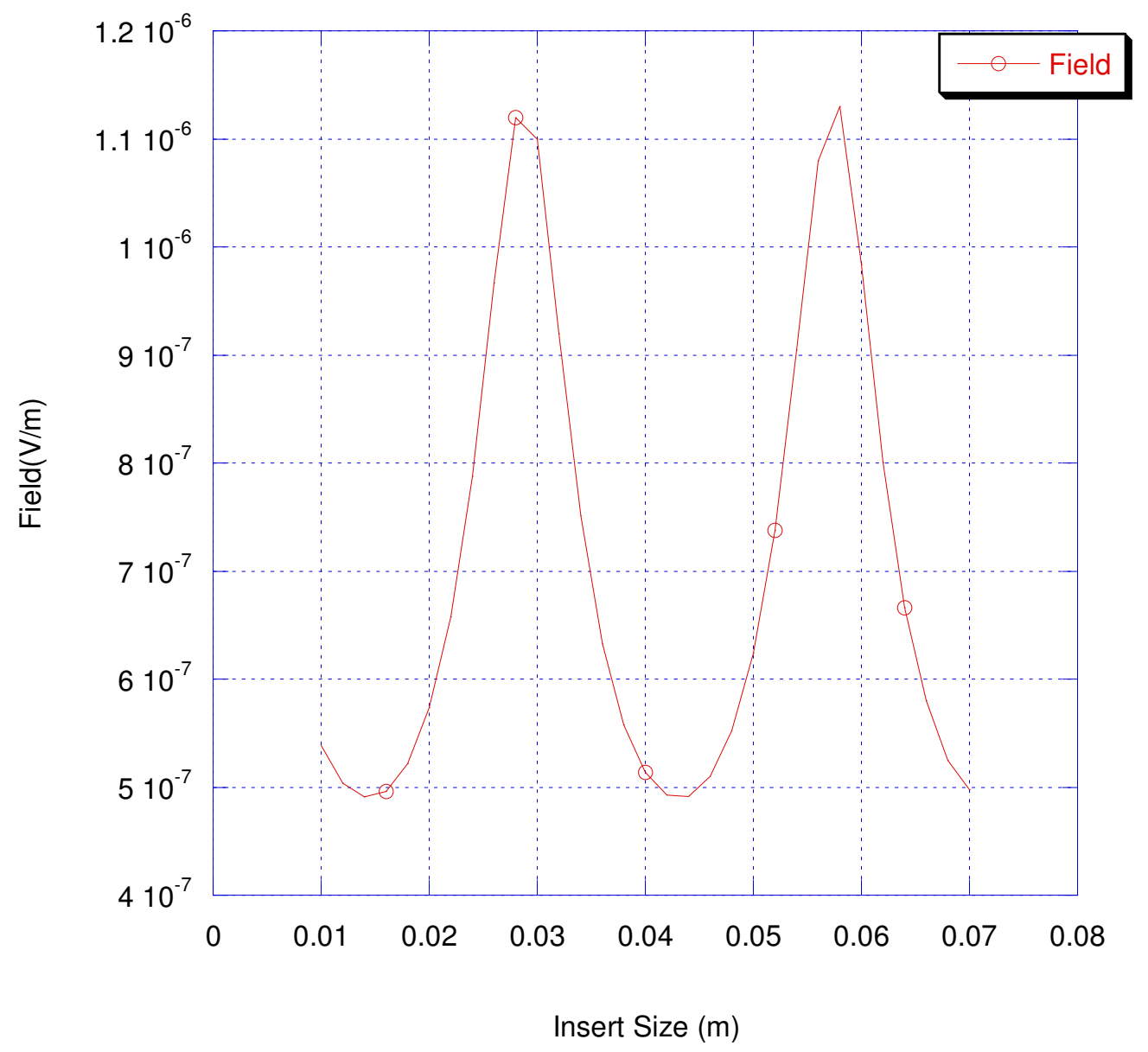

Figure 39: Electric field v/s size of dielectric insert

Selecting the size of the insert that allows a maximum for the field to exist, we get the following sizes of different components:

PTFE section: $0.0706 \mathrm{~m}$

Dielectric section: $0.026 \mathrm{~m}$ 
Air section: $0.0015 \mathrm{~m}$

Creating the model within COMSOL, we can see that the predicted results do occur, wherein an electric field does exist in the outside of the dielectric inserts, in the air. This field can also be seen to be dying out very quickly.

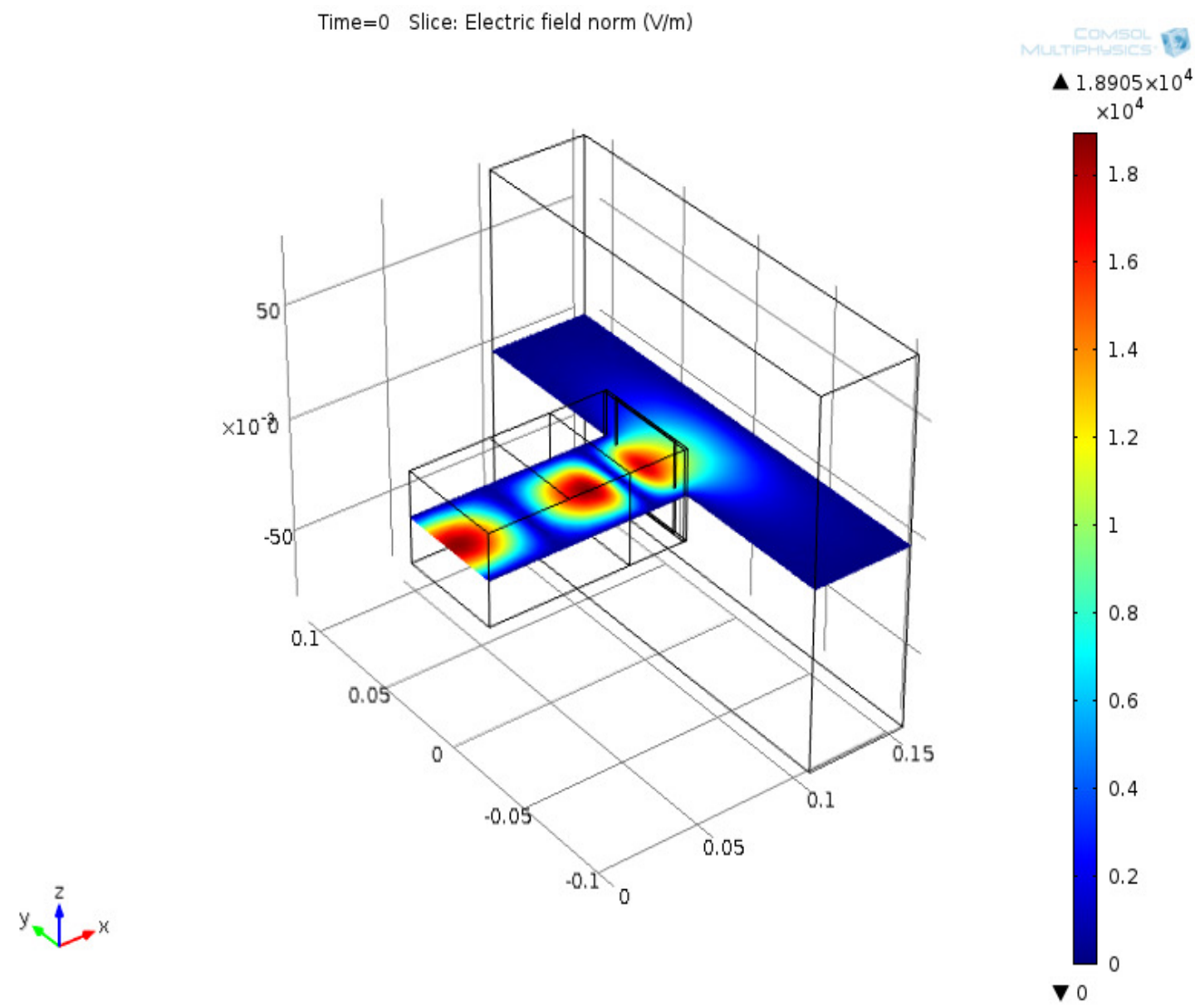

Figure 40: Field and substrate in open microwave setting

The sample to be cured was placed within this evanescent field, and the field and temperature readings for the chip are mentioned below. Taking a cut line through the 
dimension with highest expected field gradient, which would lead to the highest temperature gradient, with the cut shown below.

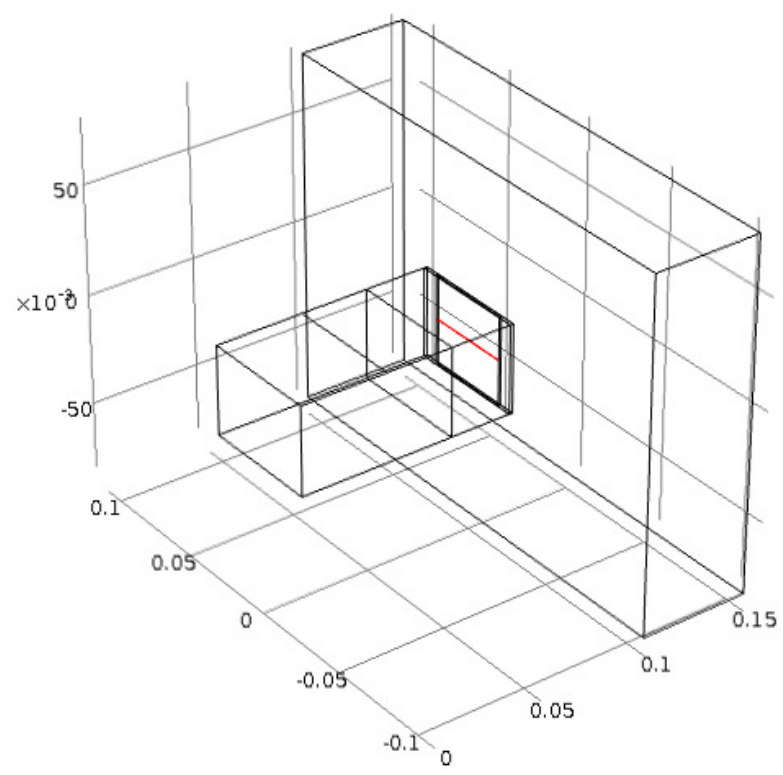

Figure 41: Cut line through the chip showing measurement points

We get an electric field pattern very similar to the chip being placed vertically in the closed cavity oven. The field intensity however is slightly higher than the closed microwave case.

This is due to the energy absorbed not contributing to the propagation loss as the wave does not propagate in this regime. 


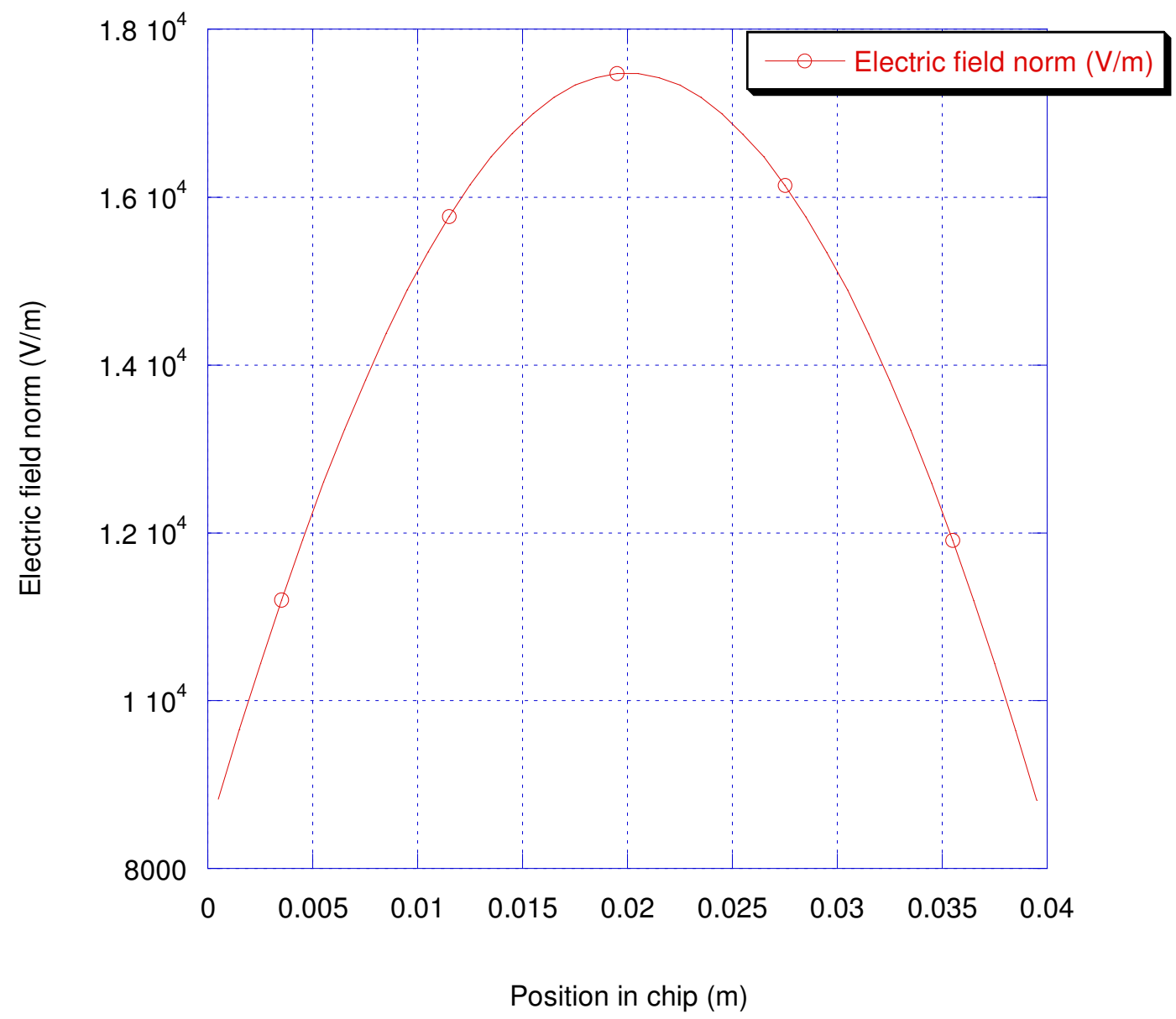

Figure 42: Electric field norm inside the chip

The similarity with the vertical placement of the substrate in a closed oven is understandable as it is the same propagation mode, and therefore the shape of the field lines would be the same. 
Heating the substrate for a short time (10 seconds), we get the temperature profile seen below.

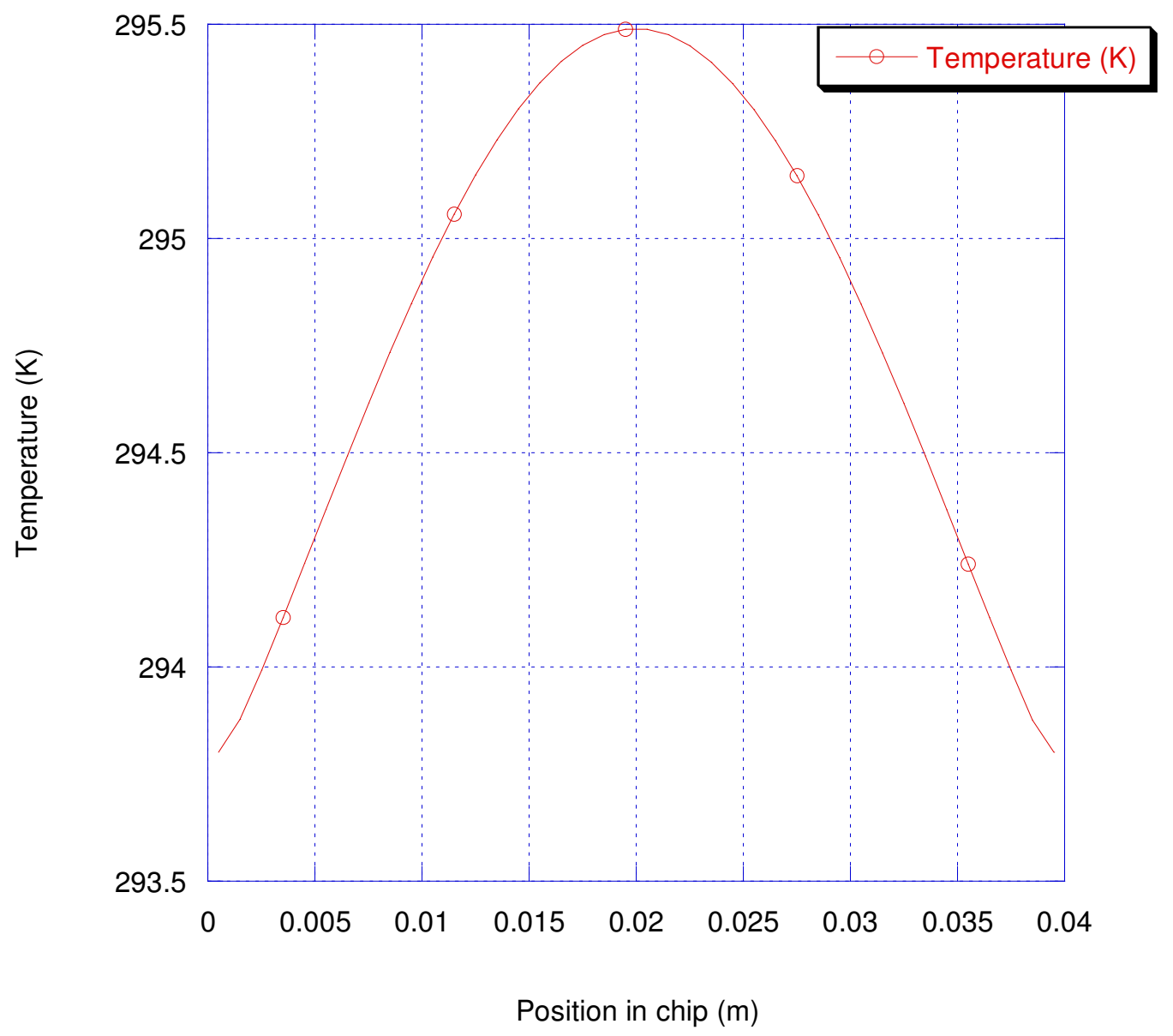

Figure 43: Temperature inside the chip after 10s

After a longer heating time, we can see the profile remaining constant as conductive heat transfer balances the microwave heating. The temperature of the cut line plotted every 250 seconds can be seen below. 


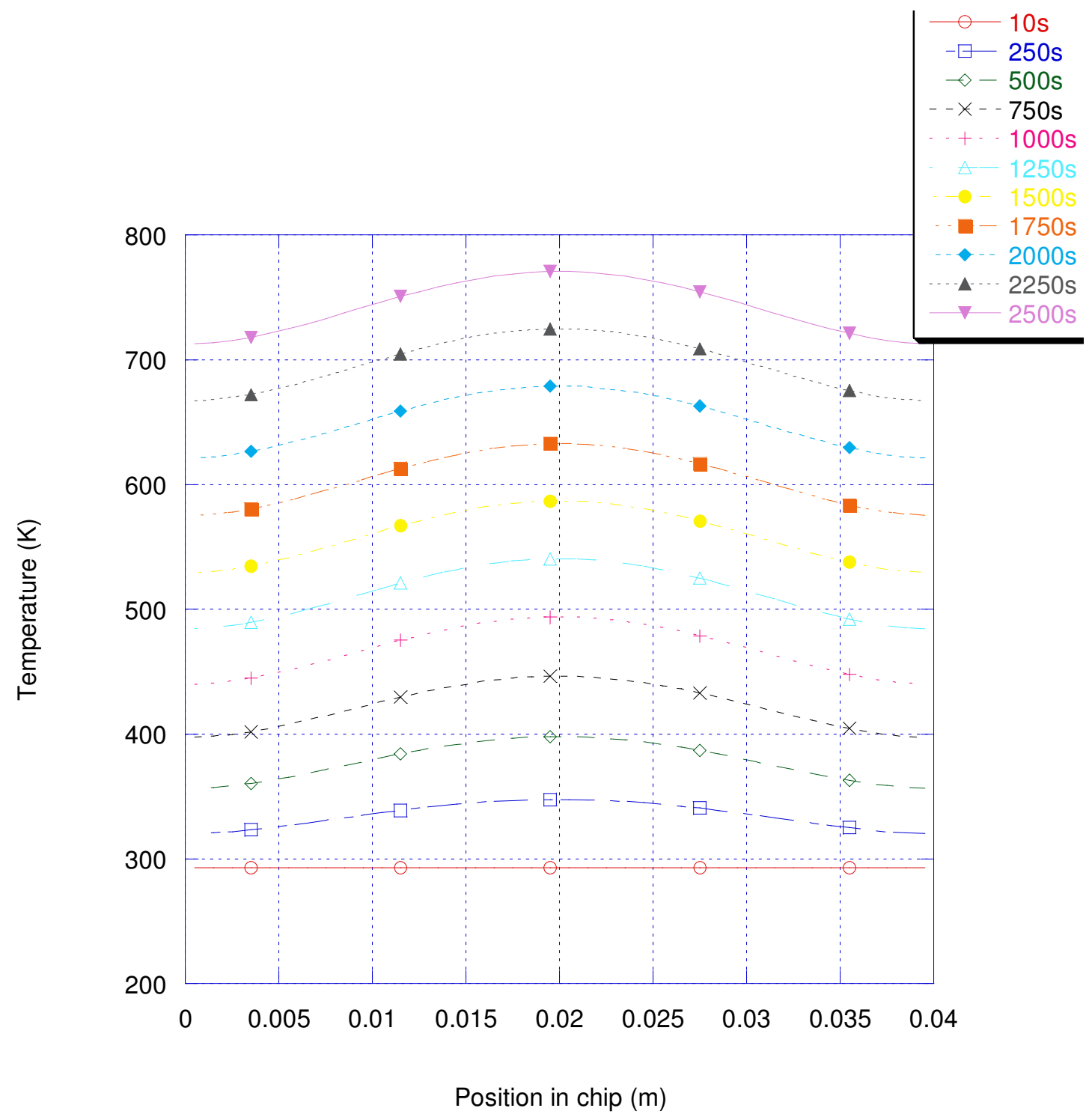

Figure 44: Temperature inside the chip at 250s intervals 


\section{Chapter 6: Conclusion}

Compiling the three cases observed in this report, we have used the same power output of $120 \mathrm{~W}$ and a single propagation mode of a single frequency. The temperature profiles of all three cases are different however. The maximum temperature in the chip plotted against time can be seen below.

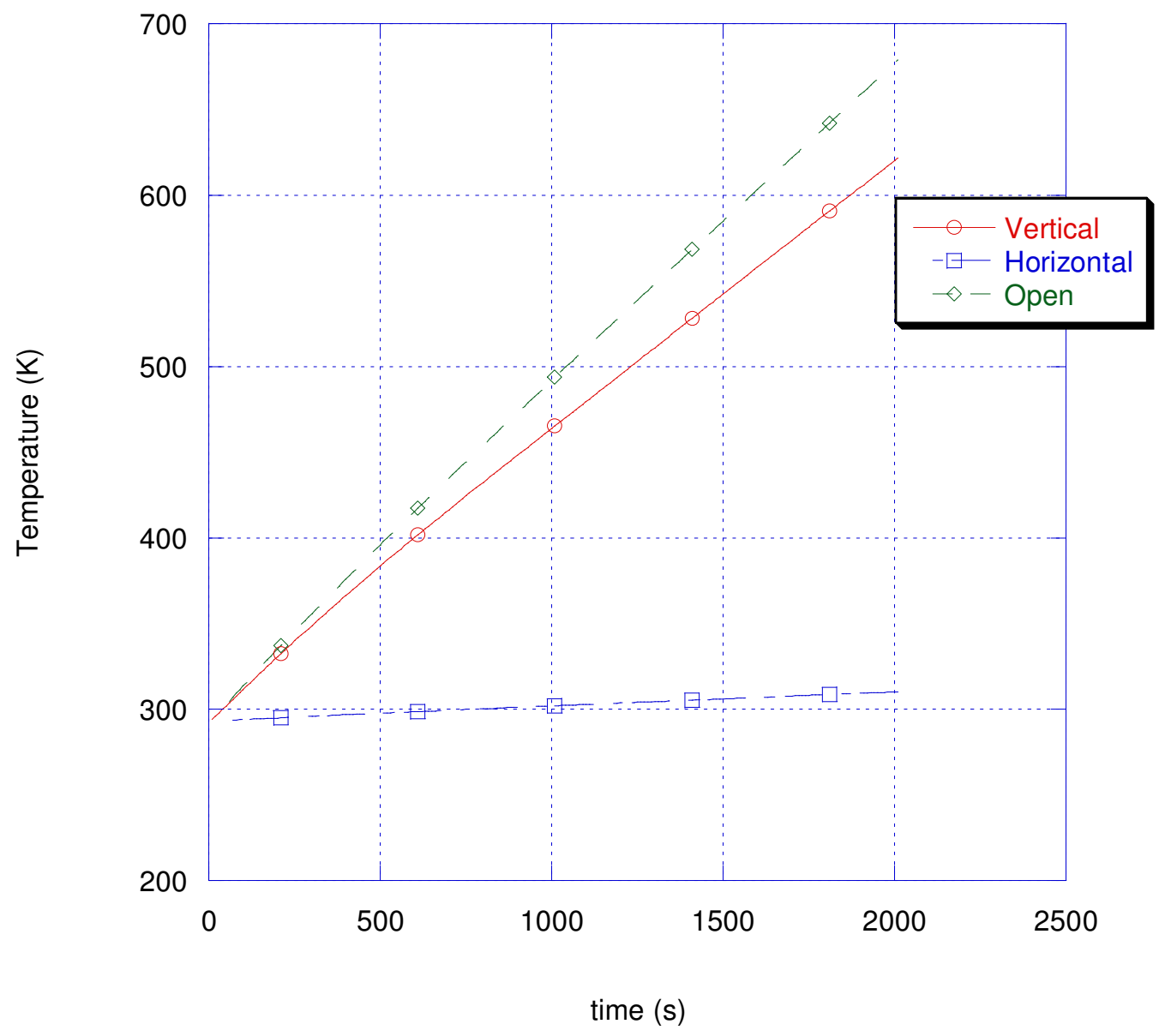

Figure 45: Maximum temperature inside each chip 
As can be seen, the open system has the highest localized increase in temperature for the inputted power. The horizontally oriented chip in the closed cavity on the other hand had the poorest result with an increase of $17.13 \mathrm{~K}$ in 2000 seconds. This increase is very small, even compared to traditional convective ovens which have ramp rates that could go upto $40 \mathrm{~K} / \mathrm{min}$ and stagnate at $473 \mathrm{~K}$ for 30 minutes. (Zhang Fan, 2000) The horizontal arrangement in the closed cavity therefore can be ruled out from the set of potential replacements for a conventional oven . The vertically oriented chip in the closed cavity however has a very similar heating rate as the open system.

The curing temperature for most polymers used in underfill applications lies in the ballpark of about $160^{\circ} \mathrm{C}$. (Chiang, 1998) This implies that orienting the chip vertically in the closed oven, or placing it near the open cavity, it would take 800 to 1000 seconds to accomplish cure. These are however localized maxima. Other edges of the substrate may not be cured at this point.

In order to interpret the results better, the Maximum temperature gradient within the chip with time is plotted below. Since the purpose of this study was to minimize the temperature gradient inside the chip, a clear development of the gradient with heating time can be observed. 


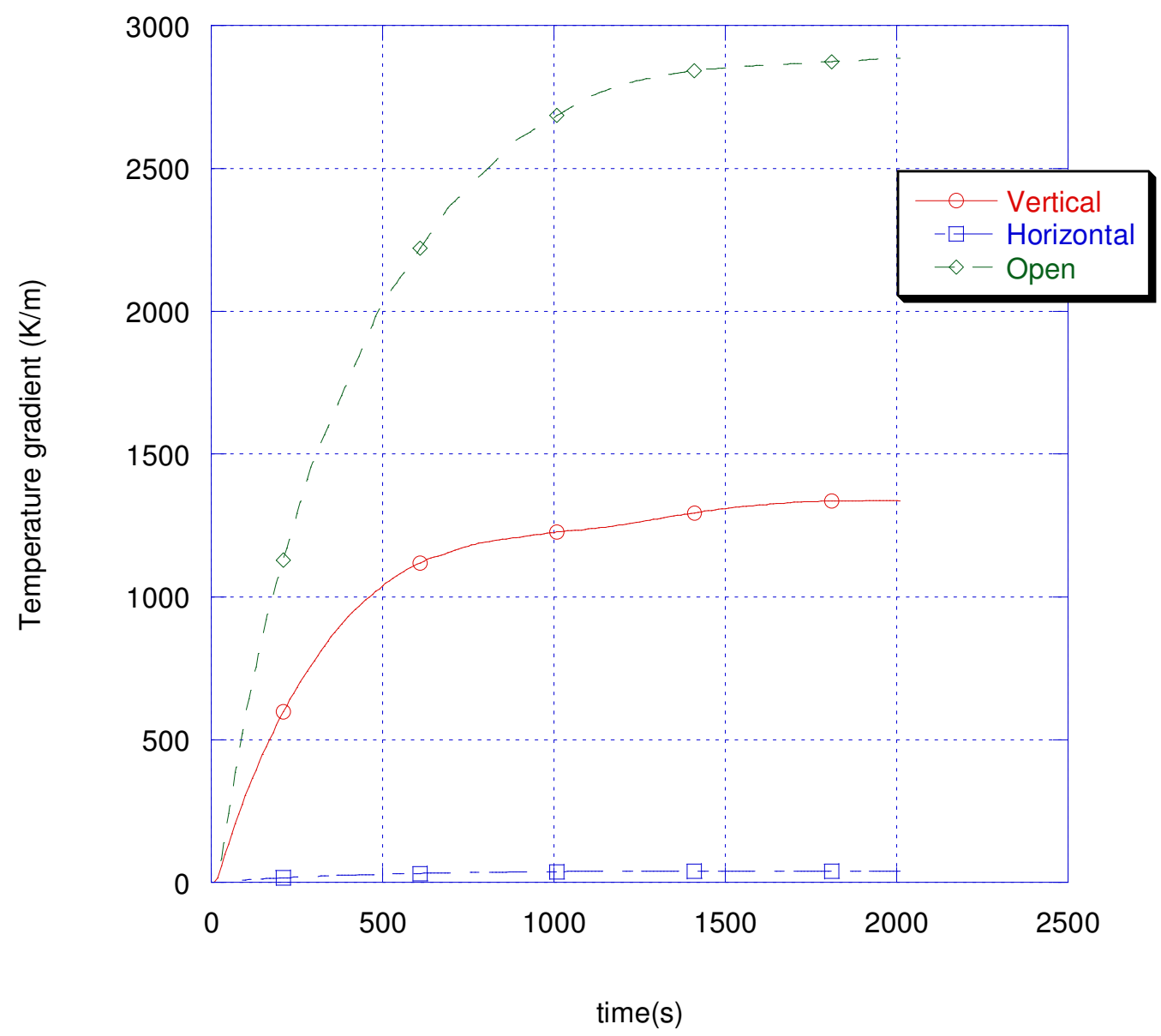

Figure 46: Maximum temperature gradient inside each chip

Expecting the sample to be cured in 1000 seconds, we can see that the gradient is still ramping up in the case of the open microwave at this point, even though it is over twice the gradient in the closed system.

Observing the temperature changes induced by the various arrangements of microwaves and their fields, we get a clear indication of the advantages of the open microwave with significant temperature increase as compared to the other cases. It however has its own 
drawback with also having a significant temperature gradient occurring in its heating process.

The other end of the spectrum has the chip placed horizontally in the TE10 closed microwave cavity. This leads to the lowest temperature gradient, however this method of heating also causes the least temperature increase overall and therefore to an extent defeats the purpose of using microwaves over conventional convection ovens for curing polymers.

The open microwave case has one other advantage wherein it can be moved and therefore the produced temperature gradient could be reduced by having the open microwave scan the chips surface. This could lead to a lower temperature gradient overall.

In a usability case however, it would be best to use a vertical placement of the substrate in a closed oven as this leads to a very similar temperature increase as the open oven case, but has a significantly lower temperature gradient through the substrate.

Lowering the power output of the magnetron would also help reduce the temperature gradient within the substrate as this would allow conductive heat transfer within the samples to regulate the temperature profile. Balancing the power output with the conductivity of the substrate would play a significant role in the mitigation of a thermal gradient, but such properties change with degree of cure for polymers and extensive material models would be required in order to conduct such studies. 


\section{Chapter 7: Future Work}

The analysis conducted during this study showed the relationship between the dimensions of the waveguide and standing wave pattern size. Using the upper limit of size as the limiting parameter, the standing wave size could be increased to $12 \mathrm{~cm}$, thus leading to a smaller energy gradient through the substrate. Such patterns could be explored in the future to improve the resultant temperature gradient.

Higher resonance modes could also be explored as by Sinclair et al. since modes such as TE11 and TM11 have a more even field distribution in planes normal to propagation modes.

Incorporating more extensive material models that consider the change in properties of the materials that are to be cured using this methodology would help paint a better picture in understanding the thermal change. 


\section{Works Cited}

Bailey, G. a. (2002). MICROWAVE CURE OF CONDUCTIVE ADHESIVES FOR FLIIP-CHIP \& MICROSYSTEMS APPLICATIONS. 2002 Inter Society Conference on Thermal Phenomena, (pp. 848-853).

Bremen. $(1997,6)$. DIELECTRIC HEATING WITH MICROWAVE ENERGY. Retrieved from Pueschner: http://www.pueschner.com/downloads/basics_adv_en.pdf

Chiang, D. C. (1998). Underfill Material Selection For Flip Chip Technology. Massachusetts: Massachusetts Institute of Technology.

Chris Pinciuc, V. M. (n.d.). simulating and modeling RF-microwave heating. Retrieved from Comsol: http://www.comsol.com/video/simulating-modeling-rf-microwaveheating

D. Salvi, D. B. (2010). Numerical Modeling of Continuous Flow Microwave Heating: A Critical Comparison of COMSOL and ANSYS. Journal of Microwave Power and Electromagnetic Energy , 187-197.

Electromagnetic Spectrum. (n.d.). Retrieved from NASA Goddard Space Flight Center: http://imagine.gsfc.nasa.gov/docs/science/know_I1/emspectrum.html

Eva Marand, K. R. (1992). Comparison of reaction mechanisms of epoxy resins undergoing thermal and microwave cure from in situ measurements of microwave dielectric properties and infrared spectroscopy. Macromolecules , 2243-2252. (2008). A Student's Guide to Maxwell's Equations. In D. Fleisch. Cambridge. Flip Chip Ball Grid Array Package Reference Guide. (2005). Texas Instruments. HISKEY, D. (n.d.). The Microwave Oven Was Invented by Accident by a Man Who was Orphaned and Never Finished Grammar School. Retrieved from Today I found Out. Johnson, R. W. Flip Chip Assembly and Underfilling. Auburn University . K.I. Sinclair, T. T. (2008). Advanced microwave oven for rapid curing of encapsulant. 2nd Electronics Systemintegration Technology Conference, (pp. 551-556). Greenwich. Martin C. Hawley, S. Z. (n.d.). Status Of Microwave Processing of Polymers and Composites. 
Mavalvala, N. W. (n.d.). 8.03 Physics III: Vibrations and Waves, Fall 2004. MIT OpenCourseWare: Massachusetts Institute of Technology.

Metaxas, A. (1991). Microwave heating . Retrieved from Pueschner: http://www.pueschner.com/downloads/MicrowaveHeating.pdf

Peter P. Silvester, a. R. (1996). Finite elements for electrical engineers. Cambridge: Cambridge University Press.

Pozar, D. M. (2012). Microwave Engineering. Massachusetts: Wiley.

Rectangular waveguide Dimensions. (n.d.). Retrieved from Microwaves101: http://www.microwaves101.com/encyclopedias/rectangular-waveguide-dimensions Staelin, D. (n.d.). 6.013 Electromagnetics and Applications, Spring 2009. MIT OpenCourseWare: Massachusetts Institute of Technology.

Sumanth Kumar Pavuluri, M. F. (2012, May). Encapsulation of Microelectronic Components Using Open-Ended Microwave Oven. IEEE TRANSACTIONS ON COMPONENTS, PACKAGING AND MANUFACTURING TECHNOLOGY, VOL. 2, NO.5 , 799805.

T. Tilford, K. S. (2007). Variable Frequency Microwave Curing of Polymer Materials in Microelectronics Packaging Applications. 9th Electronics Packaging Technology Conference, (pp. 791-796).

Wang, C. (2000). Determining Dielectric Constant and Loss . Rolla, MO: University of Missouri.

Woong Sun Lee, J. Y. (2005). Comparative study of thermally conductive fillers in underfill for the electronic components. Diamond and Related Materials , 1647-1653.

ZAK FATHI, D. T. (n.d.). INNOVATIVE CURING OF HIGH RELIABILITY ADVANCED POLYMERIC ENCAPSULANTS.

Zhang Fan, M. L.-H. (2000). An investigation into curing behavior and kinetics of underfills for flip chip packages. International Symposium on electronic materials and packaging (pp. 313-318). Institute of materials research and engineering Singapore. 